\title{
Fronto-temporal coupling dynamics during spontaneous activity and auditory processing
}

\author{
1 Francisco García-Rosales ${ }^{1 *}$, Luciana Lopez-Jury ${ }^{1}$, Eugenia Gonzalez-Palomares ${ }^{1}$, Yuranny \\ 2 Cabral-Calderín ${ }^{2}$, Julio C. Hechavarría ${ }^{1 *}$. \\ $3 \quad{ }^{1}$ Institut für Zellbiologie und Neurowissenschaft, Goethe-Universität, Frankfurt am Main, Germany. \\ $4 \quad{ }^{2}$ Research Group Neural and Environmental Rhythms, MPI for Empirical Aesthetics, Frankfurt, \\ 5 Germany. \\ 6 * Correspondence: \\ 7 Francisco García-Rosales \\ 8 garciarosales@bio.uni-frankfurt.de \\ 9 Julio C. Hechavarria \\ 10 hechavarria@bio.uni-frankfurt.de
}

12 Keywords: frontal cortex, auditory cortex, oscillations, local-field potentials, functional

13 coupling, coherence, sensory coding, auditory processing

\section{Abstract}

15 Most mammals rely on the extraction of acoustic information from the environment in order to 16 survive. However, the mechanisms that support sound representation in auditory neural networks

17 involving sensory and association brain areas remain underexplored. In this study, we address the 18 functional connectivity between an auditory region in frontal cortex (the frontal auditory field, FAF) and the auditory cortex (AC) in the bat Carollia perspicillata. The AC is a classic sensory area central for the processing of acoustic information. On the other hand, the FAF belongs to the frontal lobe, a brain region involved in the integration of sensory inputs, modulation of cognitive states, and in the coordination of behavioural outputs. The FAF-AC network was examined in terms of oscillatory coherence (local-field potentials, LFPs), and within an information theoretical framework linking FAF and AC spiking activity. We show that in the absence of acoustic stimulation, simultaneously recorded LFPs from FAF and AC are coherent in low frequencies (1-12 Hz). This "default" coupling was strongest in deep AC layers and was unaltered by acoustic stimulation. However, presenting auditory stimuli did trigger the emergence of coherent auditory-evoked gammaband activity ( $>25 \mathrm{~Hz}$ ) between the FAF and AC. In terms of spiking, our results suggest that FAF and $\mathrm{AC}$ engage in distinct coding strategies for representing artificial and natural sounds. Taken together, our findings shed light onto the neuronal coding strategies and functional coupling mechanisms that enable sound representation at the network level in the mammalian brain. 


\section{Introduction}

33 Many animals rely on the processing of acoustic information for survival. Nevertheless, the

34 mechanisms by which sounds are represented in neural networks involving distant areas in the brain

35 remain obscure. In the mammalian cortex, sensory and integration areas have been described as part

36 of putative neural networks tasked with sound processing. The auditory cortex (AC), for example,

37 plays an important role in sound analysis and even in coordinating acoustically guided behaviours

38 (Song et al., 2010; Li et al., 2017). Neuronal activity within the AC represents a large range of

39 acoustic properties including spectrotemporal structure (Gaese and Ostwald, 1995; Lu et al., 2001;

40 Yin et al., 2011; Gaucher et al., 2013; Kanold et al., 2014; Gao and Wehr, 2015; Lu et al., 2016;

41 Martin et al., 2017; Sheikh et al., 2019), sound source location encompassing azimuth/elevation

42 coding (Recanzone, 2000; Mrsic-Flogel et al., 2005; Salminen et al., 2015; Trapeau and

43 Schonwiesner, 2018) and target distance processing (Suga and O'Neill, 1979; Hechavarria et al.,

44 2013; Bartenstein et al., 2014; Beetz et al., 2016), as well as abstract properties such as sound

“emotional valence" (Concina et al., 2019) and future behavioural outcomes based on auditory

46 stimuli (Francis et al., 2018).

Regions within the frontal lobe of the mammalian brain also participate in auditory processing and could in principle synchronize their activity with that of canonical auditory areas, such as the AC (see (Winkowski et al., 2018)). Frontal and AC regions are strongly connected through feedforward and feedback anatomical pathways (Kobler et al., 1987; Medalla and Barbas, 2014; Plakke and Romanski, 2014; Winkowski et al., 2018). Neurons within the prefrontal cortex (PFC, a region in the frontal lobe) respond to sounds when the latter possess rich spectrotemporal dynamics (Eiermann and Esser, 2000; Kanwal et al., 2000; Romanski and Goldman-Rakic, 2002). Additionally, PFC is thought to engage in cognitive processes ranging from attention, learning, and memory formation/retrieval, to decision making (Miller, 2000; Floresco and Ghods-Sharifi, 2007; St Onge et al., 2011; Gourley et al., 2013; Gilmartin et al., 2014; Pezze et al., 2014; Helfrich and Knight, 2016; Kim et al., 2016; Werchan et al., 2016; Helfrich et al., 2017). This area could thus be a fundamental node for sound evaluation in auditory networks, and even for the implementation of acoustically guided behaviours.

Though there is increasing evidence supporting the idea of frontal-AC functional networks for 
LFPs) measured simultaneously in frontal and AC areas synchronize during spontaneous activity and during listening. Moreover, it is unknown whether frontal activity displays preferential coupling patterns with certain layers of the AC. Assessing the latter can only be achieved by conducting simultaneous measurements from frontal and AC regions using layer-specific intracranial recordings to study spikes and LFPs.

In the current study, we address the functional connectivity in a fronto-auditory cortical circuit in bats (species Carollia perspicillata). The bat AC has been studied extensively, and it has been shown that oscillatory and spiking activity patterns in the bat cortex are in accordance with those observed during the processing of artificial and naturalistic sounds in other animal models, including speech in humans. This comprises phenomena such as multiscale temporal processing of acoustic streams (Giraud and Poeppel, 2012; Hyafil et al., 2015; Hechavarria et al., 2016b; Teng et al., 2017; GarciaRosales et al., 2018a), interactions between spikes and LFPs for audition (Lakatos et al., 2005; Kayser et al., 2009; Arnal and Giraud, 2012; Kayser et al., 2012; Gilmartin et al., 2014; GarciaRosales et al., 2018b; Garcia-Rosales et al., 2019), and gamma-band activity for communication call processing (Medvedev and Kanwal, 2008). In bats, there exists a region within the frontal lobe that is responsive to sounds: the frontal auditory field (FAF; (Kobler et al., 1987; Eiermann and Esser, 2000; Kanwal et al., 2000)). This region is anatomically connected with the AC (Kobler et al., 1987), but it also receives auditory afferents via a non-lemniscal pathway through the suprageniculate nucleus of the thalamus, bypassing main auditory centres in the midbrain (Kobler et al., 1987). In addition to pure tones, neurons in the FAF encode spectrotemporally complex sounds with variable latencies and response properties (Eiermann and Esser, 2000; Kanwal et al., 2000; Lopez-Jury et al., 2019). Our goal was to examine specifics of fronto-AC activity in awake bats during the processing of acoustic streams. We tackled this question by quantifying neural synchronization in the FAF-AC network in terms of oscillatory coherence (a mechanism underlying interareal communication; (Fries, 2015)), during both spontaneous activity and the processing of natural and artificial acoustic sequences.

We found that the FAF-AC network is synchronized by default (i.e. without sensory stimulation) in low-frequencies (up to $12 \mathrm{~Hz}$ ), and that coherence with the FAF is strongest in deep laminae of the AC. In addition, low-frequency coherence between the two structures remains unchanged during acoustic processing, and auditory-evoked gamma-band synchronization emerges at stimulus onset without clear layer specificity. Finally, based on an information theoretical framework, our data

93 suggest that the neuronal coding of acoustic streams in FAF and AC may occur with non-overlapping 
94 neural codes. Taken together, the data presented in this manuscript offer insights into the strategies

95 for sound representation in fronto-AC neural networks.

\section{Results}

\subsection{Stimulus-related LFPs in AC lag relative to those in FAF}

98 We recorded electrophysiological data from the primary AC, paired with penetrations from the FAF, in 5 awake Carollia perspicillata bats (all males; $\mathrm{n}=50$ penetrations). Recordings in the AC were

100 performed with laminar electrodes inserted perpendicularly into the brain, spanning depths of 0-750

$101 \mu \mathrm{m}$ as measured from the cortical surface. Each penetration in AC was paired with a simultaneous

102 recording from the FAF using a single carbon electrode at an average depth of $313 \pm 56 \mu$ m (mean \pm 103 std). Auditory stimuli consisted of artificially constructed syllabic trains with repetition rates of 5.28

104 and $97 \mathrm{~Hz}$ in order to test for slow and fast periodicities in acoustic streams, plus another syllabic 105 train that had no clear rhythmicity as syllables were presented in a Poisson-like sequence with $70 \mathrm{~Hz}$ 106 average rate (see Methods). The trains consisted of a repeated short duration, broadband distress

107 syllable from $C$. perspicillata, recorded in previous work (Hechavarria et al., 2016a), whose

108 spectrotemporal design is typical of distress syllables emitted by this species. In addition, we 109 presented a natural distress vocalization ( "nat" throughout the text) that has been used in previous 110 research (Hechavarria et al., 2016b; Garcia-Rosales et al., 2018a; Garcia-Rosales et al., 2019), and

111 which comprises temporal modulations in low (ca. $4 \mathrm{~Hz}$ ) and high-frequency ranges $(>50 \mathrm{~Hz})$,

112 corresponding to the bout and syllabic periodicities, respectively, typical of this animal's distress

113 vocalizations (Hechavarria et al., 2016a).

114 Grand average traces of simultaneously recorded LFPs from FAF and AC (the latter at a depth of 450 $115 \mu \mathrm{m}$, corresponding to input layers in AC) during acoustic stimulation are shown in Fig. 1A. LFP

116 responses from frontal and auditory cortical regions showed clear modulation by the acoustic streams

117 that were well-correlated across structures for each stimulus tested, particularly at depths $>200 \mu \mathrm{m}$

118 (Fig. S1). Remarkably, population averaged LFPs appeared "faster" in the FAF than in the AC (see

119 Fig 1A, right column; blue: FAF; orange: AC at $450 \mu \mathrm{m}$ ) relative to stimulus onset. In fact, cross-

120 correlation analyses of the traces depicted in Fig. 1A showed that LFPs recorded in frontal regions

121 preceded those recorded from the AC by at least $4 \mathrm{~ms}$ (Fig. 1B), effectively indicating that primary

122 auditory cortical stimulus-related LFPs in input layers "lag" relative to those in the FAF. We

123 confirmed this trend by systematically determining the temporal lag between LFPs in the AC at 
124 various depths, and LFPs from the FAF. Figure 1C summarizes the results by illustrating, for each

125 stimulus, the distribution of lags between FAF and AC across depths (note that negative lags indicate

126 FAF "leading"). Indeed, we observed a significant effect of LFPs in the FAF being "faster" than

127 those in the AC, robust across depths in the latter structure, and also across stimuli (Fig 1C; FDR-

128 corrected tailed Wilcoxon signed rank tests, testing that the medians of the lag distributions were

129 significantly less than 0 across electrodes; $\mathrm{p}_{\text {corr }}<0.05$; precise $\mathrm{p}_{\text {corr }}$ values are indicated next to each

130 heatmap). These results were corroborated by testing FAF-AC lags in LFPs but considering only LFP

131 pairs that were well correlated across structures (correlation coefficients higher than 0.5, shown in

\section{Fig. S1).}

133 That LFPs in the frontal auditory field "lead" relative to those in the auditory cortex suggests the

134 presence of fast inputs into frontal auditory areas, agreeing with a non-lemniscal auditory pathway

135 converging into the FAF and consisting of as few as four synapses in bats (Kobler et al., 1987). We

136 sought for evidence of fast neuronal responses in FAF that would support these observations by

137 measuring response latencies of the neuronal spiking recorded simultaneously in both structures (see

138 Methods). We observed that spiking responses from the FAF could in fact be as fast as spiking

139 responses from the AC, although this effect was found only in a subpopulation of neurons (on

140 average $5.65 \% \pm 3.19 \%$ of the units considered, across stimuli and channels; see Fig. 2A, B, which

141 depicts example responses from one FAF and one AC unit at $450 \mu \mathrm{m}$ recorded simultaneously).

142 Measured neuronal response latencies from both structures yielded that AC spiking was on average

143 faster than the FAF spiking (Fig. 2C; for illustrative purposes AC responses are those recorded at

$144450 \mu \mathrm{m})$. Still, some FAF units exhibited response latencies below $10 \mathrm{~ms}$, indicative of fast acoustic

145 inputs into this structure. By subtracting latencies from simultaneously recorded units in the FAF and

146 the AC (across depths; latencies were pooled from all tested stimuli, but paring was only done within

147 a particular stimulus), it became evident that auditory cortical spiking was typically faster than its

148 FAF counterpart, although some latencies from frontal regions were shorter than those in the AC.

149 Figure 2D shows the distribution of latency differences (across cortical depths in the AC), depicting

150 the abovementioned observations. Latency differences were significantly higher than 0 for all

151 recording depths in the AC, except in the case of the most superficial channel (FDR-corrected

152 Wilcoxon signed rank tests, significance when $\mathrm{p}_{\text {corr }}<0.05$; corrected $\mathrm{p}$ values across channels are

153 given to the right of the latency distribution heatmap in Fig. 2D). 
154 Altogether, these results provide evidence supporting that the FAF receives fast auditory inputs.

155 Notwithstanding, such inputs do not necessarily elicit equally fast spiking, suggesting that the

156 neuronal dynamics in frontal areas are "sluggish" in comparison to primary AC. Sluggish dynamics

157 can arise from multiple factors, and may be essential for sensory integration in the frontal cortex (see

158 Discussion).

\section{$159 \quad 2.2 \quad$ FAF and AC synchronize in low frequency LFP bands during spontaneous activity}

160 Local-field potentials recorded in the FAF and the AC typically showed visible phase

161 synchronization, even in the absence of acoustic stimulation (see Fig. 3A, where LFP traces from

162 both structures during a single $3 \mathrm{~s}$ epoch of spontaneous activity are depicted). We quantified phase

163 coherence between the two structures by means of the imaginary part of the coherency ("iCoh" in

164 this manuscript; see Methods and (Nolte et al., 2004)), for data recorded both during spontaneous and

165 sound-driven activities. The iCoh metric allows to minimize spurious phase-synchrony attributable,

166 for example, to common referencing and passive spreading of field potentials, by effectively

167 removing non-lagged phase correlations (Bastos and Schoffelen, 2015).

168 Coherence analyses revealed that, as shown in Fig. 3A, FAF and AC were synchronized in low

169 frequencies. Figure 3B depicts population averaged z-normalized (to a surrogate distribution where

170 phase relationships were abolished; see Methods) iCoh values (z-iCoh) across electrode depths in the

171 AC. Elevated low-frequency coherence is evident in deep layers of the AC, suggesting as well that

172 FAF-AC synchrony was depth-dependent. A time-resolved analysis of iCoh (Fig. 3C) over the same

173 LFP traces used to calculate values in Fig. 3B also showed that low-frequency phase synchrony was

174 strongest in deeper channels, and furthermore limited to the low-frequency region of the coherence

175 spectrum. The data depicted in Fig. 3C are shown here for illustrative purposes and serve as a

176 comparison with the time-resolved coherence estimations performed on LFPs recorded during

177 acoustic processing (see below).

178 To statistically corroborate our observations, we divided the coherence spectrum into canonical

179 frequency bands encompassing delta (1-4 Hz), theta (4-8 Hz), alpha (8-12 Hz), beta (12-25 Hz) and

180 low-gamma $(25-45 \mathrm{~Hz})$. We then tested if z-normalized iCoh values in each band were significantly

181 different than 0 across the population. Because of the nature of the surrogate analyses (see Methods),

182 non-consistent phase synchronization in the data would yield a distribution of z-iCoh statistically

183 indistinguishable from 0 . In order words, z-iCoh values significantly higher than 0 suggest consistent, 
184 population-wise phase-locking between LFPs in FAF and AC during spontaneous activity. Figure

$1853 \mathrm{D}$ (top) depicts the z-iCoh calculated between FAF and AC oscillations for each frequency band at

186 various AC depths. For frequency bands between 1-12 Hz (i.e. delta to alpha), we observed

187 significantly higher than $0 \mathrm{z}$-iCoh estimates (FDR-corrected Wilcoxon signed rank tests, $\mathrm{p}_{\text {corr }}<0.05$

188 for significance; log-converted p values are shown in Fig. 3D, bottom), which became gradually

189 lower towards higher frequencies (note also the decay in Fig. 3B). Beta or gamma z-iCoh

190 distributions were not significantly different than 0 at any cortical depth.

191 It was also apparent that coherence values were depth dependent in the AC, particularly in the delta

192 band (where z-iCoh was strongest, see Fig. 3B, D). We tested the depth dependence of coherence by

193 comparing the distributions of z-iCoh for all pairs of channels, across all penetrations and frequency

194 bands. Results are summarized in significance matrices depicted in Fig. 3E. Each cell $(i, j)$ in a

195 matrix represents the log-converted, corrected p value (FDR Wilcoxon signed rank tests), obtained

196 after statistically comparing FAF-AC coherence using an AC channel at depth $i$, and another at depth

$197 j$. In the matrices, cells within red contour lines correspond to statistically significant $\mathrm{p}_{\text {corr }}$ values ( $\mathrm{p}_{\text {corr }}$

198 < 0.05). As shown in Fig. 3E, deep electrodes in the AC were significantly better synchronized with

199 the FAF, an effect only visible in the delta band.

\subsection{FAF-AC synchronization during acoustic processing}

201 To quantify synchronization during acoustic processing in the FAF-AC circuit, we calculated time-

202 resolved iCoh values in response to four acoustic stimuli (a natural call, syllabic trains of 5.28 and 97

$203 \mathrm{~Hz}$, and a syllabic train with Poisson temporal structure; see above). Population average coherograms

204 (i.e. time-frequency representations of iCoh values) are shown in Fig. 4A-D for all stimuli tested, and

205 at representative depths in the $\mathrm{AC}(50,450$ and $700 \mu \mathrm{m})$. The most conspicuous pattern across

206 stimuli was the appearance of low-gamma coherence (typically in the range of 25-45 Hz), which was

207 associated with stimulus onset (at time 0) and apparently independent of auditory cortical depth.

208 Gamma synchrony was auditory-evoked, a notion strengthened when considering responses to the

$2095.28 \mathrm{~Hz}$ syllabic train (Fig. 4B), where the evoked coherence tracked individual syllable

210 presentations. Remarkably, we observed little evidence for an increase of low-frequency synchrony

211 when compared to spontaneous activity (compare heatmaps in Fig. 4 with Fig. 3C). That is, even

212 though low-frequency coherence was present before sound presentation (in line with our results using

213 spontaneous LFPs), it did not change visibly after stimulus onset. To illustrate the occurrence of low

214 and high frequency coherence, Figure 4E-H depict single-trial LFPs from a representative pair of 
215 simultaneous penetrations in the AC (450 $\mu \mathrm{m}$ depth) and FAF. Raw LFPs are shown in their

216 broadband form $(0.1-300 \mathrm{~Hz}$ ), and filtered in frequency ranges of 4-12 Hz and 25-45 Hz (i.e. low and

217 high frequencies oscillations). The range 4-12 Hz was chosen for low-frequency activity because

218 spectral parameters at lower frequencies could not be reliably estimated with the window size chosen

219 for time-resolved coherence analyses (200 ms; see Methods). Note that the occurrence of

220 synchronized waves in the AC and FAF is clear after stimulus onset (0-ms mark).

221 The defined low- and high-frequency ranges were then used to quantify changes from spontaneous to 222 stimulus-driven coherence in the FAF-AC network. A systematic, time-resolved analysis of low-

223 frequency synchrony revealed that, across stimuli and auditory cortical depths, there was little change

224 (calculated as percentage increase from spontaneous to sound-driven activity: $\left[\mathrm{iCoh}_{\text {stim }}-\mathrm{iCoh}_{\text {spont }}\right] /$

$225 \mathrm{iCoh}_{\text {spont }} * 100$ ) in coherence between both structures (Fig. 5A, D, G, J, top heatmaps). The data

226 showed that low frequency synchrony preceded stimulus presentation in deep layers, seldom reached

$22750 \%$ increase from spontaneous across stimuli (black contour lines in Fig. 5A, D, G, J), and when it

228 did, it typically happened at middle AC depths. We did not observe statistical evidence showing

229 significant increase of low frequency coherence at stimulus onset (i.e. first $100 \mathrm{~ms}$ after sound

230 presentation; Fig. 5B, E, H, K, black traces; FDR-corrected Wilcoxon signed rank tests, significance

231 when $\left.\mathrm{p}_{\text {corr }}<0.05\right)$.

232 Figure 5C, F, I, L (top heatmaps) illustrate effect size calculations (r; see Methods) for the low-

233 frequency coherence increase in a time resolved manner across channels. In the heatmaps, only time

234 points where the increase was significantly different from 0 (uncorrected Wilcoxon singed-rank test,

$235 \mathrm{p}<0.05$ ) are shown. From this analysis the following was evident: (i) the pattern of significance was

236 inconsistent across stimuli for low frequency coherence; and (ii) effect sizes were typically small,

237 with areas of medium effect size (those within grey contour lines) appearing also with an inconsistent

238 pattern across sounds. Large effect sizes (within red contour lines) were overall only observed in

239 small clusters, lacking consistency throughout the stimulus set. The boundaries between small,

240 medium and large effect sizes were defined as follows: $r<0.3$, small; $0.3 \leq r<0.5$, medium; $r \geq 0.5$,

241 large (Fritz et al., 2012). Altogether, these results corroborate a lack of reliable increase in low-

242 frequency coherence between FAF and AC during passive listening, compared to spontaneous

243 activity.

244 High frequency FAF-AC coherence was considerably more sensitive to acoustic stimulation. As

245 expected from the data depicted in Fig. 4, we observed a strong increase of low-gamma interareal 
246 synchronization associated to the stimulus onset (Fig. 5A, D, G, J, bottom heatmaps). Stimulus-

247 evoked gamma synchrony was typically higher than 50\% (reaching values as high as $80 \%$ ) and

248 tracked the syllable presentations of the $5.28 \mathrm{~Hz}$ syllabic train (Fig. 5D, bottom). Indeed, the increase

249 of low-gamma synchrony at stimulus onset was significantly above 0 for all stimuli (Fig. 5B, E, H,

$250 \mathbf{K}$, grey traces; same statistical analysis as for low-frequencies; $\mathrm{p}_{\text {corr }}<0.05$ ), and more reliably so for

251 channels located in input layers of the AC (in this context, electrodes at depths of 250-350 $\mu \mathrm{m}$; layers

252 III-IV in C. perspicillata's AC span depths of 200-450 $\mu \mathrm{m}$, see (Garcia-Rosales et al., 2019)). For the

$2535.28 \mathrm{~Hz}$ and the Poisson syllabic trains, the onset-related increase in low-gamma coherence occurred

254 essentially along all AC depths studied. In terms of effect size (Fig. 5C, F, I, L; bottom heatmaps),

255 we observed large effects of increased low-gamma coherence at stimulation onset across stimuli (less

256 clearly in the case of the natural vocalization), with sustained, seemingly periodic increases along the

257 time-course of the $5.28 \mathrm{~Hz}$ syllabic sequence. Taken together, coherence analysis results indicate that

258 acoustic stimulation elicits auditory-evoked low-gamma synchronization between the frontal auditory

259 field and the auditory cortex.

\section{$260 \quad 2.4$ Gamma-band activity in FAF and AC}

261 Previous studies showed the occurrence of gamma-band activity in the AC of primates, bats, and rats

262 (Brosch et al., 2002; Medvedev and Kanwal, 2008; Vianney-Rodrigues et al., 2011). These studies

263 reported auditory cortical gamma which was not time-locked to the onset of a stimulus, and that

264 appeared even hundreds of milliseconds after sound presentation. Given the nature of the coherence

analyses performed here, it is possible that the presence of non-locked gamma oscillations could have

266 been overlooked in the FAF and the $\mathrm{AC}$ of $C$. perspicillata. To explore the occurrence of these

267 rhythms in our dataset, we focused on the onset period of the $5.28 \mathrm{~Hz}$ syllable train as it was the

268 stimulus that permitted the analysis of an onset window without the influence of subsequent sounds

269 (after the first syllable presentation), for a sufficiently long time-lapse of at least $180 \mathrm{~ms}$. The time

270 period around the first syllable presentation in the $5.28 \mathrm{~Hz}$ train was subdivided into three segments:

271 (1) a window of $90 \mathrm{~ms}$ spanning times before stimulus onset (pre); (2) a window of $90 \mathrm{~ms}$ starting at

272 stimulus onset (onset); (3) a window of $90 \mathrm{~ms}$ starting $90 \mathrm{~ms}$ past stimulus onset (late); and (4) a

273 window of $180 \mathrm{~ms}$ starting at stimulus onset ( $f u l l$ ). The span of these segments is illustrated in Fig.

274 6A together with representative LFP traces from a penetration pair. The segments were chosen in

275 order to contrast LFP power at different frequency bands (low frequencies, 0-15 Hz; low-gamma, 25 - 
$45 \mathrm{~Hz}$; high-gamma 45-80 Hz; and broad gamma 25-80 Hz) with spontaneous LFP power before stimulus presentation (pre window).

There was a consistent increase of onset-related gamma activity in FAF and AC, particularly during the onset period, which was potentially linked to an evoked activation in cortex as it was associated with a broadband increase in LFP power (see Fig. 6B, green traces, and Fig. S2A). To uncover the presence of gamma within later time periods in our data, the power of different frequency bands in the late period was statistically compared (Wilcoxon signed rank test, significance threshold at $\mathrm{p}=$ 0.01; see Methods) with the power in the pre period on a trial-by-trial basis, per penetration (Fig. 6C). The percentage of penetrations in FAF where there was a power increase in low frequencies was of $8 \%$, reaching between $14-24 \%$ in the AC. We also observed a relatively small number of penetrations $(<10 \%)$ either in AC or FAF in which there was a significant power increase in gamma for the late period as compared to the pre segment. We further calculated the time course of the percentage of penetrations showing significant power increase at times surrounding the stimulus presentation for both FAF and AC, at various LFP frequencies (Fig. 6D; see Methods). As expected from the data shown in Fig. 6B-C and Fig. S2A, up to about $50 \mathrm{~ms}$ after stimulus onset there was a high percentage of penetrations (ca. 80\%; cf. with Fig. S2A) where the power in gamma increased significantly in either structure. This number was relatively low $(<20 \%)$ for times beyond 50-60 ms after stimulus onset.

The FAF-AC circuit exhibited increased auditory-evoked gamma band coherence, related to the onset of acoustic stimulation (Figs. $\mathbf{4 , 5}$ ). We tested to what extent we could disentangle gamma activity in our data from a non-specific broadband response, by means of previously used approach which relies on comparing the relative power distributions of gamma and low frequency LFPs (Medvedev and Kanwal, 2008). In this case, evidence for the gamma-band activity being a different component from the broadband evoked-related potentials relies on the statistical independence between low- and highfrequency power in the LFPs. For this analysis the full window was used (see Fig. 6A). The power distributions of gamma (either $25-45$ or $45-60 \mathrm{~Hz}$ ) and low frequencies typically did not differ in our dataset (see Fig. 7A, E for a representative penetration). A systematic population analysis was performed to quantify the percentage of penetrations in the data for which there was evidence of statistical independence between the power of gamma and that of low-frequency potentials. As depicted in Fig. 7B, F, power distributions of gamma (in the 25-45 and 45-60 Hz ranges) and low 
tests, significance when $\mathrm{p}<0.01)$ only in a small proportion $(<15 \%)$ of the total amount of

penetrations, either in FAF or AC. This could suggest that gamma-band activity in these frequency ranges cannot be readily disentangled from a broadband power increase related to an onset response, assuming that if they were separable processes (i.e. gamma and low frequency activities) their power distributions would differ significantly (Medvedev and Kanwal, 2008).

312 We reasoned, however, that a lack of significant differences between the distributions does not

313 necessarily imply that the relative powers of gamma and low-frequencies are well correlated when

314 considering trial specific information. Strong correlations would occur if low and high frequency

315 relative powers were tightly determined by the strength of the broadband activation. Therefore, given

316 a weak correlation, it could be argued that the dynamics of low- and high-frequency (gamma) LFPs

317 might be more complex than an unspecific power increase related to the evoked response. We

318 observed poor correlations, across penetrations in FAF and AC, between low-frequency and gamma-

319 band relative power on a trial-by-trial basis (see, for example, Fig. 7C, G). The distribution of

320 correlation coefficients for the population data is depicted in Fig. 7D, H. Overall, correlation

321 coefficients were low, having a median in the FAF of $0.22\left(25^{\text {th }}\right.$ and $75^{\text {th }}$ percentiles: 0.12 and 0.35$)$

322 for the $25-45 \mathrm{~Hz}$ gamma range, and of $0.07\left(25^{\text {th }}\right.$ and $75^{\text {th }}$ percentiles: -0.05 and 0.23$)$ for the $45-60$

$323 \mathrm{~Hz}$ band. In the AC, the median across channels was of 0.17 ( $25^{\text {th }}$ and $75^{\text {th }}$ percentiles: 0.03 and 0.31 )

324 for the $25-45 \mathrm{~Hz}$ gamma, and of $0.08\left(25^{\text {th }}\right.$ and $75^{\text {th }}$ percentiles: -0.04 and 0.1$)$ for the band of 45-60

325 Hz. Typically, no more than 20-25\% of the penetrations in AC and FAF showed a significant

326 correlation (significance when $\mathrm{p}<0.01)$ between relative power at low-frequencies and gamma $(25-$

$32745 \mathrm{~Hz}$, median $20 \%$ of sites; $45-60 \mathrm{~Hz}$, median $8 \%$ of sites; see Fig. S2B).

328 We also quantified how the overall energy of early activation correlates with the gamma coherence

329 increase in the FAF-AC circuit. Gamma-band coherence increase (compared to spontaneous activity,

330 see Fig. 5) was poorly correlated with evoked potential energy for all AC channels (median across

331 channels: $0.15 ; 25^{\text {th }}$ and $75^{\text {th }}$ percentiles: 0.08 and 0.22), as illustrated in Fig. 7I for representative

332 depths in AC, and in Supplementary Fig. S3 for all depths. Although gamma-band activity is not

333 straightforwardly separable from a broadband activation pattern, the data shown in Fig. S3 and the

334 poor trial-by-trial correlation between gamma and low-frequency powers (see above) suggest the

335 possibility of interesting gamma-band dynamics in C. perspicillata's FAF and AC. 
337 We investigated spike-spike interactions in the FAF-AC network within an information theoretical

338 framework (Shannon, 2001). Mutual information (MI or "information" throughout the text) between

339 the stimuli and neuronal responses allows to quantify the theoretical ability of a neuron (or a set

340 thereof) to represent the acoustic input on a single trial basis (see Methods). MI captures all non-

341 linear dependencies of any statistical order in the data, and its quantification depends on the neural

342 code being considered (Kayser et al., 2009). Here, we aimed to determine the coding abilities in AC,

343 FAF, and a joint response from both structures based on a spike rate code $\left(\mathrm{I}_{\mathrm{rate}}\right.$ for single units, $\mathrm{I}_{\text {joint }}$

344 considering responses from AC and FAF together; (Kayser et al., 2009; Garcia-Rosales et al.,

345 2018a)). A rate code was considered so that our results could be comparable with previous data

346 obtained from C. perspicillata's AC (Garcia-Rosales et al., 2018a).

347 Overall, we relied on an information theoretic approach because we observed that representations in

$348 \mathrm{AC}$ and FAF were quite different and on occasions, at least in appearance, complementary. For

349 example, Fig. 6A depicts spiking from two simultaneously recorded FAF and AC units in response to

350 the natural stimulus. Note how the firing rate of the FAF unit increases as the stimulus progresses,

351 whereas the AC unit is time-locked to slow temporal modulations in the stimulus and does not

352 respond like its FAF counterpart. Information theory would allow to measure possible interactions

353 between these responses in a quantitative manner.

354 Figure 6B shows a schematic of the rate code used to quantify MI for single units and joint

355 responses. For subsequent analyses and in order to guarantee that all units considered were auditory-

356 responsive (both in $\mathrm{FAF}$ and $\mathrm{AC}$ ), we used only responses that provided at least $0.1 \mathrm{bit} / \mathrm{s}$ of

357 information (with a window size of $4 \mathrm{~ms}$ this corresponds to $4 \times 10^{-3}$ bits; see Methods). The number

358 of units used to quantify $\mathrm{I}_{\text {rate, }}$, per channel (including the FAF electrode, and across the AC linear

359 probe) is depicted in Fig. 6C, left. Note that the number of penetrations is equivalent to the number

360 of units per channel in this case: $n \geq 13$ in FAF, and $n \geq 29$ in AC. For paired responses (FAF-AC)

361 the number of units considered was less because the inclusion criterion ( $\left.\mathrm{I}_{\mathrm{rate}} \geq 0.1 \mathrm{bit} / \mathrm{s}\right)$ had to be

362 fulfilled by units in AC and FAF simultaneously ( $\mathrm{n} \geq 8$ pairs; Fig. 6C, right).

363 Population values of $\mathrm{I}_{\text {rate }}$ for the FAF and the AC at different depths are depicted in Fig. 6D, for each

364 stimulus. Two main conclusions can be drafted from this figure. (i) In the AC, the highest

365 information about the stimuli was found at depths between 200-650 $\mu$ m. (ii) Neurons in the FAF

366 were on average less informative than AC ones, but were well above the limit set in the inclusion

367 criterion across stimuli (nat: $0.56 \pm 0.09 \mathrm{bits} / \mathrm{s}, 5.28 \mathrm{~Hz}$ train: $0.45 \pm 0.06 \mathrm{bits} / \mathrm{s}, 97 \mathrm{~Hz}$ train: $0.48 \pm$ 
0.04 bits/s, Poisson train: $0.42 \pm 0.05$ bits/s; given as mean \pm s.e.m). We also quantified the information provided by paired neuronal responses from both regions ( $\left.\mathrm{I}_{\text {joint }}\right)$, which is illustrated as black traces in Fig. 6E. This figure also shows a direct comparison between $\mathrm{I}_{\text {joint }}$ and the $\mathrm{I}_{\text {rate }}$ of FAF

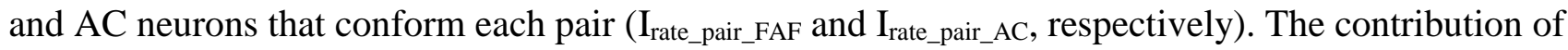

$372 \mathrm{I}_{\text {rate }}$ from the FAF was significantly smaller than the contribution of $\mathrm{I}_{\text {rate }}$ from the AC (FDR-corrected

373 Wilcoxon signed rank tests, $p_{\text {corr }}<0.05$; corrected $p$ values of all comparisons are given in Fig. S4). Although $\mathrm{I}_{\text {rate }}$ in the FAF was always $>0.1 \mathrm{bit} / \mathrm{s}$, we did not observe $\mathrm{I}_{\text {joint }}$ to be significantly higher than the $I_{\text {rate }}$ from the AC ( $I_{\text {rate_AC; }}$ orange traces in the Fig. 6E), across electrodes and stimuli tested (Fig. S7). We did observe $\mathrm{I}_{\text {joint }}$ to be well correlated with the sum of $\mathrm{I}_{\text {rate }}$ calculated using the information of FAF and AC spiking $\left(I_{\text {sum }}=I_{\text {rate_pair_FAF }}+I_{\text {rate_pair_AC }}\right.$; Figs. S5-S9).

When considering the interactions of spiking across structures in terms of the codes defined in this study, the linear relationship between $\mathrm{I}_{\text {sum }}$ and $\mathrm{I}_{\text {joint }}$, evident in Figs. S5-S9, would support the notion of "independence" in the information provided by the spiking in each structure. Independence arises theoretically when $\mathrm{I}_{\text {joint }}=\mathrm{I}_{\text {sum }}$, and implies that each structure represents different aspects of the sensory stimulus. However, the results illustrated in Fig. 6E suggest that independence cannot be inferred from the data with certainty. Mathematically, independence would require $\mathrm{I}_{\text {joint }}$ to be higher than both $\mathrm{I}_{\text {rate }}$ in AC and FAF simultaneously, which was not fulfilled at a population level: $\mathrm{I}_{\text {joint }}$ was not significantly different than $\mathrm{I}_{\text {rate }}$ obtained from AC units (see Fig. S4). Calculating information estimates using time windows of up to $12 \mathrm{~ms}$ yielded comparable results, indicating that the temporal resolution of the codes had little effect in the described outcomes (data not shown). Overall, our quantification of stimulus-related spiking information, occurring simultaneously in the AC and FAF, suggests different sound coding strategies in these two structures. These observations are further addressed in the Discussion section.

\section{Discussion}

In this study we investigated the functional connectivity between frontal and auditory cortical regions of the bat Carollia perspicillata. Specifically, we examined the coupling dynamics of the FAF, a frontal area which receives auditory afferents from cortical and subcortical structures, and the primary AC. Functional connectivity was assessed during spontaneous activity and during the processing of natural and artificially generated acoustic sequences. Our main results are: i) LFPs recorded simultaneously in both regions suggest that the FAF receives faster auditory inputs relative to the $\mathrm{AC}$, yet these inputs do not necessarily elicit faster spiking in the FAF; ii) during spontaneous 
activity, the FAF-AC network is coupled in low frequencies (up to $12 \mathrm{~Hz}$ ), with stronger coherence values at deep layers of the AC; iii) while acoustic stimulation does not considerably alter the default low-frequency coupling, auditory-evoked gamma-band synchronization in the FAF-AC circuit emerges upon sound presentation; iv) considering a spiking rate code, the FAF is less informative than the $\mathrm{AC}$ about the acoustic stimuli, while a joint code using simultaneous spiking from both regions suggests that FAF and AC engage in distinct coding dynamics. Altogether, our data shed light onto how distant brain areas in the mammalian brain engage in sound representation. The results of this paper are summarized in Fig. 9.

\subsection{Auditory afferents into the FAF}

Stimulus-related LFPs recorded simultaneously from FAF and primary AC, at various depths in the latter structure, indicate the presence of fast synaptic inputs into the frontal region that precede those arriving into the AC even at input layers. The work of Kobler, Casseday, and colleagues in the late 1980s (Kobler et al., 1987; Casseday et al., 1989), showed that the FAF receives auditory afferents via a non-canonical pathway that bypasses major auditory centres in the midbrain, including the inferior colliculus (IC). In this pathway, acoustic information from neurons in the cochlear nucleus is

414 sequentially relayed to the anterolateral olivary complex, the suprageniculate nucleus of the thalamus (SGN), and from there into the FAF. Thus, although auditory inputs reach the frontal region also through the AC, acoustic information may reach the frontal field, directly from the cochlea, in as few as four synapses (Kobler et al., 1987). Synaptic currents related to inputs from the SGN into the FAF could lead to changes in LFPs (Buzsaki et al., 2012) faster than their counterparts at input layers of the $\mathrm{AC}$, which are predominantly driven by thalamocortical synapses originating in the ventral region of the medial geniculate body. Therefore, a rapid, non-canonical pathway into FAF accounts for our observations regarding the temporal relation between LFPs in both regions studied.

422 The results described in this manuscript indicate that, although sound-related LFPs from the FAF 423 lead those from the AC, the neuronal spiking latencies are shorter in AC. In other words, our data 424 indicate that faster inputs in FAF are not sufficient to elicit faster spiking in most cases.

425 Electrophysiological studies in frontal auditory areas have shown that neuronal responses are usually of relatively large latencies (although short latencies are also to be found), sparse and of high

427 variability (Newman and Lindsley, 1976; Eiermann and Esser, 2000; Kanwal et al., 2000; Plakke and 428 Romanski, 2014). Recent data from C. perspicillata's FAF highlighted the possibility that the 
and long-lasting synaptic dynamics, at least considering projections from the AC (Lopez-Jury et al., 2019). These slow, long-lasting synaptic dynamics could support sensory integration, by conditioning FAF neurons to spike after accumulating synaptic inputs over time, and/or to integrate multiple synaptic inputs originating from different sensory modalities. Certainly, cross-modal sensory

434 integration occurs in the frontal cortex (Fuster et al., 2000; Romanski, 2007; Hwang and Romanski, 2015), whereas integration over relatively long timescales appears to be a feature of higher-order cortical areas in general (Runyan et al., 2017).

Fast acoustic afferents, even without eliciting reliable stimulus-evoked spiking, imply nonetheless that auditory information is already present in the FAF before it receives inputs from the AC. Within the predictive coding framework (Arnal and Giraud, 2012; Friston, 2018), it is then possible to hypothesize that the frontal field may be a region where prediction errors could also be generated.

That is, the rapid non-lemniscal pathway could relay faithful information into FAF about the auditory stimuli, which can in turn be compared with information received from primary AC and the along the auditory hierarchy (Carbajal and Malmierca, 2018), and generated in the SGN and the AC, are integrated in the FAF. The result of such integration could in turn be used to update the “expectations" of the system. In rodents, prediction error signals appear all along the auditory pathway, but occur more strongly in higher-order structures (Parras et al., 2017), while prediction error signals related to sounds have also been described in the frontal cortex of rats (Imada et al., 2012) and humans (Durschmid et al., 2016; Durschmid et al., 2018). The involvement of the FAF in predictive coding could be thoroughly tested in future experimental work.

A number of FAF neurons project directly to the superior colliculus (SC), a structure related to motor control of head and pinna movement in bats (Kobler et al., 1987). The presence of early acoustic information in FAF, the fact that frontal neurons in $C$. perspicillata show preference for naturalistic echolocation acoustic stimuli (high-frequency pulses used to navigate by bats; (Eiermann and Esser, 2000)), and the existence of projections from FAF into motor-related structures such as the SC, suggest that the FAF plays an important role in coordinating auditory-guided behaviour. This would be in line with proposed roles of prefrontal cortex in motor control (Risterucci et al., 2003), including volitional motor (vocal) production after acoustic stimulation (Hage and Nieder, 2015). However, 
461 We examined the default (i.e. in the absence of external stimulation) functional connectivity in the

462 FAF-AC network in terms of oscillatory coherence (Fig. 3). The data presented in this manuscript

463 show that simultaneously recorded LFPs from both structures are phase-synchronized in low-

464 frequencies of the spectrum (1-12 Hz), although more strongly so in the delta band (1-4 Hz).

465 Empirical evidence points towards a role of oscillatory coherence in the coupling of distant brain

466 regions, a view that is summarized in proposed theoretical mechanisms such as the communication-

467 through-coherence framework (Fries, 2015). Several studies have pinpointed an involvement of low-

468 frequency synchronization in the functional coupling between the frontal cortex and a variety of brain

469 structures. Coherent oscillations between distant cortical areas (including the frontal cortex) in the

470 low-frequency range correlate with working memory (Daume et al., 2017), fear memory

471 consolidation (Popa et al., 2010), attentional selection (Womelsdorf and Everling, 2015), and long-

472 term fear recall (Cambiaghi et al., 2016; Karalis et al., 2016). In the auditory domain, top-down

473 control exerted from frontal cortical areas, through low-frequency oscillatory activity, increases

474 coupling to speech signals in the human AC (Park et al., 2015). Our results indicate that, in the bat

475 brain, frontal areas that participate in audition are functionally interconnected by means of low-

476 frequency LFPs with primary auditory cortex. Critically, such coupling does not require external

477 input, which hints towards the presence of a default synchrony in the fronto-auditory cortical

478 circuitry. The latter could constitute a functional basis for high-order, interareal auditory processing

479 in the mammalian brain.

480 Because recordings in the AC were performed with a laminar probe, we were able to study the

481 laminar dependence of FAF-AC synchrony in AC. During spontaneous activity, low-frequency

482 coherence was strongest in deep layers (depth $>700 \mu \mathrm{m}$ ), but the strength of coherence was only

483 affected by depth in the delta band. Interestingly, a recent study revealed that, also during

484 spontaneous activity, spike-LFP synchronization was strongest in deep layers of the AC (Garcia-

485 Rosales et al., 2019). Such spike-LFP coupling was associated to the presence of discrete

486 spontaneous states of increased spiking rate (UP-states) in laminae V and VI. The origins of deep

487 layer UP-states are unclear, but it has been hypothesized that they could be driven by higher-order

488 structures (Sakata and Harris, 2009). In the current study, we observed a putative higher-order

489 auditory structure (the FAF in the frontal lobe) synchronized via a low-frequency oscillatory channel

490 with deep layers of a primary sensory area (the AC). From the phase correlation of delta-band LFPs

491 in the FAF-AC network, it is possible to speculate that delta oscillations in FAF could modulate UP- 
states in AC. However, we note that causality cannot be inferred from our current dataset and needs

to be addressed thoroughly with further experimental approaches.

In all, the "default" coupling in the FAF-AC circuit is supported by the presence of anatomical connections between frontal and auditory cortices (Kobler et al., 1987)). Although it has to be properly addressed, we propose that homologous frontal regions tasked with audition in other species may be functionally interconnected with $\mathrm{AC}$ in a similar manner. This possibility is still unexplored, yet addressing this question might be crucial for unravelling the mechanisms of high-order auditory processing, cognition, and behaviour based on audition.

\subsection{Functional coupling in the FAF-AC network during acoustic processing}

501 Functional coupling between FAF and AC was also addressed in the context of acoustic processing (Figs. 4, 5). Animals were exposed to four distinct acoustic streams, including a conspecific distress vocalization, and three "trains" constructed by repeating a distress syllable at distinct rates.

504 Independently of the stimulus used, we observed little change in the low-frequency phase synchrony of the FAF-AC network, as compared to spontaneous activity. This is a puzzling result which, assuming that low-frequency oscillatory coupling in the network is useful for auditory perception, could in principle be explained largely by our experimental design. Although the animals were awake during the experiments, they listened to the sequences passively: i.e. they were not expected to behave in response to the stimulus, and other variables (e.g. attentional processes) were not modulated according to a controlled experimental approach. Thus, statistically negligible and unreliable changes in the low-frequency dynamics of FAF-AC connectivity could be explained by the fact that the passive listening of acoustic streams is not sufficient to alter the default functional coupling in the network. Whether attention (a top-down process) or behavioural planning could modify the neuronal connectivity in the circuit by either enhancing it or decreasing it, as compared to spontaneous activity, needs to be tested in further research.

516 Experimental evidence suggests that oscillatory activity in the gamma range is crucial for neuronal

517 computations, including sensory processing and cognitive mechanisms (Fries, 2009). Previous

518 studies have shown the presence of gamma-band activity in primary auditory cortex of rats (Vianney-

519 Rodrigues et al., 2011), monkeys (Brosch et al., 2002) and bats (Medvedev and Kanwal, 2008)

520 during passive listening. These studies reported the presence of gamma oscillations at even later time 521 periods (150-300 ms) in what could be considered "induced" (as opposed to "evoked") activity, not- 
522 locked to the auditory stimulus. Our results show that, past $90 \mathrm{~ms}$ and up to $180 \mathrm{~ms}$ after stimulus

523 presentation, such late, non-locked gamma oscillations are relatively scarce ( $\sim \%$ of out of 50

524 penetrations). These results should not be taken as evidence for the lack of late gamma oscillations in

525 the AC or FAF of C. perspicillata, because later periods (190-300 ms after sound presentation) in

526 which oscillatory activity may have occurred were not analysed due to the nature of the stimulus

527 (note that the second syllable presentation of the $5.28 \mathrm{~Hz}$ occurs already at $~ 189.4 \mathrm{~ms}$ ). Further

528 research can be aimed at detecting gamma activity at later time points in the cortex of $C$.

529 perspicillata.

530 In humans, sources of auditory-evoked gamma-band activity (aeGBA) can be found both in primary

531 auditory and frontal (anterior cingulate cortex, ACC) cortices (Mulert et al., 2007; Polomac et al.,

532 2015). Frontal aeGBA is modulated by attention and correlates with performance in auditory

533 detection tasks (Debener et al., 2003; Gurtubay et al., 2004). Moreover, aeGBA in the frontal lobe

534 and gamma-band synchronization between frontal and auditory cortical regions correlate too with

535 task difficulty (Mulert et al., 2007; Polomac et al., 2015), suggesting a role of gamma-band

536 coherence in a fronto-auditory cortical circuit for cognitive control in audition. In fact, disorders of

537 the central nervous system such as schizophrenia are marked by a dysregulation of aeGBA in frontal

538 regions (Cho et al., 2006; Leicht et al., 2010; Curic et al., 2019), further supporting the importance of

539 gamma-band activity for cognition.

540 Our data indicate that low-gamma $(25-45 \mathrm{~Hz})$ coherence in the FAF-AC circuit significantly

541 increases with sound presentation, independently of the stimulus considered. This supports a role of

542 gamma synchronization between frontal and auditory cortices for auditory processing, although the

543 functional significance of aeGBA and its coherence across cortical areas should be considered with

544 care. Coherent activity does not imply directly that there exists effective communication between two

545 given regions. Moreover, the nature of the gamma activity is also to be examined cautiously. Here we

546 attempted to disentangle gamma oscillations from a frequency unspecific power surge related to

547 auditory evoked responses (Fig. 7), which would in principle explain gamma coherence between

548 FAF and AC. Based on a method proposed by Medvedev and Kanwal (2008), we did not observe

549 statistical evidence supporting that the distributions of low-frequency and gamma-band LFP power

550 were significantly different from one another across penetrations in FAF and AC (Fig. 7B, F).

551 However, we did observe, across multiple penetrations, a lack of trial-by-trial correlation between the

552 LFP power in the abovementioned frequency bands (Fig. 7C, D, G, H, and Fig. S2B). In addition, 
our data also showed a very weak correlation between the energy of the evoked response in the LFP and the gamma-band coherence increase (Fig. 7I and Fig. S3). While these results do not conclusively demonstrate that gamma-band activity can be separated from a frequency unspecific onset response, they hint towards the possibility of evoked gamma activity being an important component for audition, as suggested by previous work (Brosch et al., 2002; Medvedev and Kanwal, 2008; Vianney-Rodrigues et al., 2011). The functional roles of onset-related gamma activity in the FAF-AC circuit of $C$. perspicillata (and in the mammalian auditory system in general) should be thoroughly addressed with dedicated experimental approaches in the future.

We would like to note that it remains speculative what might be potentially signalled by the FAF-AC gamma synchronization reported in this study. Short onset-related coherence increase might convey information about the presence of an acoustic stimulus, but not necessarily allow to elucidate the stimulus' spectrotemporal features. In the AC of the bat Pteronotus parnelii, Medvedev and Kanwal (2008) reported that the spectral properties of the gamma component of the response could be used to differentiate among a battery of conspecific communication calls. In primary visual cortex, for example, distinct characteristics of stimulus-induced gamma rhythms (e.g. peak frequency or amplitude) encode for distinct properties of presented visual stimuli (e.g. contrast or orientation; (Hermes et al., 2015; Murty et al., 2018)), although it has been argued that the variability of gamma based on stimulus properties may constrain the utility of the rhythm for complex integrative computations, at least in early visual cortex (Henrie and Shapley, 2005; Ray and Maunsell, 2010; Bartoli et al., 2019). Our stimulus set is not ideal to determine in an unbiased manner whether the nature of FAF-AC gamma synchronization changes given the spectrotemporal characteristics of the stimuli, in particular because LFPs synchronize to a stimulus' temporal structure also in the gamma range (see (Hechavarria et al., 2016b; Garcia-Rosales et al., 2018a). The latter could alter the spectral patterns of coherence without necessarily meaning that the nature of the underlying coherence is changing, which is an artefact that needs to be controlled for. The careful and systematic variation of acoustic properties of sounds could be used in further research to explore in full the patterns of FAFAC gamma-band coherence and its role for audition.

\subsection{Mutual information in the FAF-AC circuit}

581 Quantifying the amount of information provided by FAF and AC about the acoustic stimuli revealed 582 that the former structure was, in comparison, significantly less informative than the latter. In addition, when considering responses from frontal or auditory cortices in a joint rate code, it was not possible 
to determine a clear population trend towards independence, redundancy or synergy between the spiking activities of both structures, from an information theoretic perspective. We propose two candidate explanations for our results, which need not be mutually exclusive. First, it is possible that the way in which FAF encodes incoming auditory stimuli is not sufficiently well-captured by means of a code based on spiking rate, and therefore the true encoding capabilities could be underestimated by assuming such scheme (see (Masquelier, 2013; Insanally et al., 2019)). Second, we note that the fact that the FAF conveys, in comparison, significantly less information than the AC, is an expected result as the $\mathrm{AC}$ is a structure specialized for auditory computations. As part of the frontal cortex, it is plausible that the FAF encodes for other variables that go beyond acoustic features (e.g. ethological relevance of the sound, multimodal sensory information, etc.). In that case, FAF units would yield low $I_{\text {rate }}$ values when attempting to quantify their abilities to encode a sound based on relatively simple methodological approaches, which rely solely on acoustic processing. The former allows to hypothesize that FAF and AC might engage in distinct, non-overlapping coding strategies.

\section{$4 \quad$ Methods}

\subsection{Animal preparation and surgical procedures}

All experimental procedures were performed in compliance with current German and European

600 regulations on animal experimentation. Experiments were approved by the Regierungspräsidium Darmstadt (experimental permit \#FU-1126). The study was performed on 5 adult bats of the species Carollia perspicillata (all males). Animals were obtained from a colony in the Institute for Cell Biology and Neuroscience, Goethe University, Frankfurt am Main. Bats used for experiments were kept separately from the main colony.

Before undergoing surgical procedures, bats were anaesthetized with a mixture of ketamine (10 $\mathrm{mg}^{*} \mathrm{~kg}^{-1}$, Ketavet, Pfizer) and xylazine (38 $\mathrm{mg}^{*} \mathrm{~kg}^{-1}$, Rompun, Bayer). For surgery and any subsequent handling of the wounds, local anaesthesia (ropivacaine hydrochloride, $2 \mathrm{mg} / \mathrm{ml}$, Fresenius Kabi, Germany) was applied subcutaneously in the scalp area. A rostro-caudal midline incision was made in the scalp, after which skin and muscle tissues were removed carefully in order to expose the skull. A sufficiently large area of the bone was also exposed to make possible the attachment of a

611 custom-made metal rode (1 $\mathrm{cm}$ length, $0.1 \mathrm{~cm}$ diameter), used during recordings to fixate the

612 animal's head. The rod was attached with dental cement (Paladur, Heraeus Kulzer GmbH, Germany).

613 Animals were given at least one full day of recovery after surgery, and before experiments were 
614 performed upon them. The AC and the FAF were located based on well-establish landmarks such as

615 blood vessel patterns, and the sulcus anterior (see (Esser and Eiermann, 1999; Eiermann and Esser,

616 2000)). On the first day of recordings each cortical region was exposed by cutting a small hole $(\sim 1$

$617 \mathrm{~mm}^{2}$ ) in the skull with a scalpel blade.

618 Recordings, which lasted no more than 4 hours a day, were performed chronically on awake bats.

619 Water was given to the animals at a period of approximately 1-1.5 hours. Between recording

620 sessions, a bat was allowed to recover for at least a full day. Experiments for the day were halted if

621 the bat showed any sign of discomfort.

\section{$622 \quad 4.2$ Electrophysiological recordings}

623 Recordings were made inside an electrically isolated and sound-proofed chamber. Inside the

624 chamber, bats were placed upon a custom-made holder which was kept at a constant temperature of

$62530^{\circ} \mathrm{C}$ with a heating blanket (Harvard, Homeothermic blanket control unit). A speaker (NeoCD 1.0

626 Ribbon Tweeter; Fountek Electronics, China), located inside of the chamber $12 \mathrm{~cm}$ away from the

627 animal's right ear (contralateral to the hemisphere were recordings were performed), was used for

628 free-field stimulation. The speaker was calibrated using a 1/4-inch microphone (Brüel \& Kjær, model

629 4135, Denmark), which was connected to a custom-made amplifier.

630 Data were acquired from the bat's left AC as described in a previous study (Garcia-Rosales et al.,

631 2019). Neurophysiological data were recorded from the AC using 16-channel laminar electrodes

632 (Model A1x16, NeuroNexus, MI; impedance: 0.5-3 M 2 ), with a channel separation of $50 \mu \mathrm{m}$. The

633 probe was carefully inserted into the brain perpendicular to the cortical surface using a piezo

634 manipulator (PM-101, Science 455 products GmbH, Hofheim, Germany) until the top-channel was

635 barely visible on the surface of the tissue. Thus, we were able to record from depths ranging 0-750

$636 \mu \mathrm{m}$, reaching all layers in AC. Histological confirmation of the extent of the electrodes inside the

637 cortex are detailed elsewhere (Garcia-Rosales et al., 2019). Recordings were made in primary AC,

638 although we cannot discard the presence of columns from high frequency fields (Esser and Eiermann,

639 1999). The laminar probes were connected to a micro-amplifier (MPA 16, Multichannel Systems

$640 \mathrm{MCS}$ GmbH, Reutlingen, Germany), and acquisition was done via a portable multichannel system

641 with integrated analogue-to-digital converter (Multi Channel Systems MCS GmbH, model ME32

642 System, Germany) with a sampling frequency $20 \mathrm{kHz}$ and a precision of 16 bits. Data acquisition was 
643 on-line monitored and stored in a computer using MC_Rack_Software (Multi Channel Systems MCS

$644 \mathrm{GmbH}$, Reutlingen, Germany; version 4.6.2).

645 For recordings in the FAF, a single carbon electrode (Carbostar-1, Kation scientific; Impedance at 1 $646 \mathrm{kHz}$ : 0.4-1.2 M $\Omega$ ) was inserted into the frontal region of the left hemisphere and lowered to depths

647 of $\sim 300-450 \mu \mathrm{m}$ with the aid of a second piezo manipulator (same characteristics as the previous

648 one). The electrode was connected to a micro-amplifier which was also connected to the integrated

649 multichannel recording system as described above. It was possible to use the same hardware because

650 the integrated system accommodates up to 32 simultaneous channel recordings. Ground and

651 reference electrodes (silver wires) were inserted as to only touch the dura mater of non-auditory

652 regions of the bat's brain, preferentially located in occipital areas of the contralateral hemisphere.

\subsection{Acoustic stimulation}

654 Acoustic stimulation was controlled from the recording computer using a custom-written Matlab 655 (version 7.9.0.529 (R2009b), MathWorks, Natick, MA) software. As acoustic stimuli we used a 656 natural distress call from $C$. perspicillata and three synthetic trains constructed from a single distress

657 syllable, repeated at different rates. Procedures for recording the natural sequence are described in a 658 previous study (Hechavarria et al., 2016a). The call is representative of $C$. perspicillata's vocal 659 repertoire, and has been used by us in previous studies addressing auditory processing at the level of 660 the AC (Hechavarria et al., 2016b; Garcia-Rosales et al., 2018a; Garcia-Rosales et al., 2019). Distress 661 calls of $C$. perspicillata exhibit two prominent, coexistent temporal modulations: the syllabic- and 662 bout rhythmicities. Syllabic rates in C. perspicillata's distress utterances are in the range of $>30 \mathrm{~Hz}$ 663 (median, 71.4 Hz; iqr: 57.1 Hz), whereas bouts (groups of syllables emitted in close sequence) are 664 repeated with rates typically $<12 \mathrm{~Hz}$ (Hechavarria et al., 2016a). In the natural call used here, 665 syllables are repeated on average with a rate of $63.7 \mathrm{~Hz}$ (see (Garcia-Rosales et al., 2018a)), whereas 666 bouts are uttered with a rate of $4 \mathrm{~Hz}$ (i.e. 8 bouts in $1.96 \mathrm{~s}$ ).

667 To emulate the temporal dynamics of the communication sequences, a stereotypical distress syllable 668 was used to construct artificial acoustic sequences. A first syllabic train had a repetition rate of 5.28 $669 \mathrm{~Hz}$, matching the slow temporal dynamics of $C$. perspicillata's distress utterances. A second one, 670 with a repetition rate of $97 \mathrm{~Hz}$, was used to simulate fast temporal dynamics in communication 671 streams. Finally, we constructed a syllabic train where syllables were repeated in a Poisson-like 672 manner, with an average rate of $70 \mathrm{~Hz}$. This simulated fast-repetition rates without any periodicity 
673 and without a slow temporal structure. The 5.28 and $97 \mathrm{~Hz}$ trains had a duration of $2 \mathrm{~s}$, while the

674 Poisson train was $4 \mathrm{~s}$ long. The syllables had an intensity of $70 \mathrm{~dB}$ SPL (root-mean square), close to

675 the intensity of the natural call (see (Garcia-Rosales et al., 2018a)).

676 Sounds were digital-to-analogue converted by means of a sound card (M2Tech Hi-face DAC, 384

$677 \mathrm{kHz}, 32 \mathrm{bit}$ ) and amplified (Rotel power amplifier, model RB-1050) in order to be presented through

678 the speaker inside of the chamber. Prior to presentation the call and syllabic trains were down-

679 sampled to $192 \mathrm{kHz}$, and low-pass filtered ( $80 \mathrm{kHz}$ cut-off). All sounds were pseudorandomly

680 presented 50 times each, with and inter-stimulus interval of $1 \mathrm{~s}$. A period of $300 \mathrm{~ms}$, and another of

$681500 \mathrm{~ms}$, was appended at the beginning and the end of each sequence, respectively.

682 Prior to any acoustic stimulation, per penetration, electrophysiological data were acquired for a 683 period of $180 \mathrm{~s}$. These data were used for coherence analyses during spontaneous activity.

\subsection{Separation of spiking activity and local-field potentials}

685 All analyses were performed offline with custom-written Matlab (version 8.6.0.267246 (R2015b))

686 scripts. Initially, the raw electrophysiological signal from each channel (all electrodes in AC and

687 FAF) was bandpass filtered (fourth-order Butterworth filter) in order to extract traces pertaining

688 spiking activity (300-3000 Hz cut-off frequencies) and LFPs (0.1-300 Hz cut-off frequencies). For

689 computational reasons, LFP data were down-sampled to $1 \mathrm{kHz}$ and stored for subsequent analyses.

690 Spike detection and sorting from FAF and AC electrodes were performed using the SpyKING

691 CIRCUS toolbox (Yger et al., 2018). Spike detection threshold was set at 5 median absolute

692 deviations from the noise baseline, and spike sorting was done automatically by the SpyKING

693 CIRCUS algorithm based on the probe's geometry to avoid detecting the same templates in two

694 adjacent electrodes. Each template was assigned to the electrode where its amplitude was the

695 strongest. Per electrode (either in AC or FAF), we chose as representative spiking the template with

696 the highest spike count. Spiking responses relative to a single electrode are referred to as a "unit" in

697 the manuscript.

\subsection{Spike latency estimation}

699 Spike latency was defined as the time point in which a unit's spiking rate was statistically different

700 from the expected rate during spontaneous activity, based on a previous study (Chase and Young,

701 2007). In brief, the algorithm proposed by Chase and Young compares a unit's response to a stimulus 
across several time windows, with the expected spiking rate under the assumption that the unit fires spontaneously with Poisson statistics, given a certain rate. A unit's firing rate in the $250 \mathrm{~ms}$ silence period before stimulus onset, across the 50 repetitions from all stimuli tested (a total 200 trials), was considered its spontaneous spiking rate for the abovementioned assumption. The response of a unit to a certain stimulus (i.e. spiking after stimulus onset) was pooled across trials. Taking this pooled response, the probability of observing at least $n$ spikes in a given window $t_{n}$ (after stimulus onset), assuming Poisson firing in the absence of acoustic inputs, can be defined as follows (Chase and Young, 2007):

$$
P_{t_{n}}(\geq n)=1-\sum_{m=0}^{n-1} \frac{\left(N \lambda t_{n}\right)^{m} e^{-N \lambda t_{n}}}{m !}
$$

711 where $\mathrm{N}$ is the number of repetitions of the given stimulus, and $\lambda$ is the spontaneous firing rate.

712 Starting from stimulation onset, the probability that each elicited spike indicates a stronger than

713 chance deviation in rate from the firing rate estimated in the absence of stimulation (the $250 \mathrm{~ms}$

714 window), is taken as the probability that the spontaneous firing rate would have produced that

715 particular spike as the last of $n$ spikes in a window $t_{n}$. In this context, $t_{n}$ is the width of the window

716 containing the $n$ spikes observed so far. Hence, the time of the first spike for which the

717 aforementioned probability is sufficiently low (here, $P_{t_{n}}(\geq n)<10^{-5}$ ) is considered as the unit's

718 latency. This method circumvents caveats regarding classical peak latency estimations using peri-

719 stimulus time histograms or spike-density functions over time (Levakova et al., 2015).

\section{$720 \quad 4.6$ Interareal coherence analyses}

721 All coherence analyses were done using the Chronux toolbox (Bokil et al., 2010). As a metric of

722 interareal phase synchronization we used the imaginary part of the coherency ("iCoh" in the

723 manuscript; (Nolte et al., 2004)), both during spontaneous activity and acoustic processing.

724 Coherency is complex value that measures phase consistency between two time series, across several

725 trials. The coherency between two signals $x$ and $y$, at a certain frequency $\omega$, can be defined as follows

726 (Bastos and Schoffelen, 2015):

$$
\operatorname{coh}_{x y}(\omega)=\frac{\frac{1}{n} \sum_{k=1}^{n} A_{x}(\omega, k) A_{y}(\omega, k) e^{i\left(\phi_{x}(\omega, k)-\phi_{y}(\omega, k)\right)}}{\sqrt{\left(\frac{1}{n} \sum_{k=1}^{n} A_{x}^{2}(\omega, k)\right)\left(\frac{1}{n} \sum_{k=1}^{n} A_{y}^{2}(\omega, k)\right)}}
$$


where $A_{x}(\omega, k)$ and $A_{y}(\omega, k)$ are the amplitudes of signals $x$ and $y$ at frequency $\omega$ and trial $k$, while $\operatorname{coh}_{x y}$ represents the coherency between both signals, and $n$ is the number of trials per stimulus $(\mathrm{n}=$ 50). Coherency is a complex quantity, but its absolute value ranges from 0 to 1 , indicating the relative, normalized strength of phase synchronization between time series. Taking the imaginary part of $\operatorname{coh}_{x y}$ is a straightforward manner to remove non phase-lagged interactions that could be attributable to, for example, passive field spread or common referencing (Bastos and Schoffelen, 2015). In order to minimize further common influences related to the temporal structure of the stimuli, we subtracted from each trial the mean (across all trials of a given stimulus) LFP of each channel (Kikuchi et al., 2017), and calculated coherence using the de-meaned traces. Note that this could affect low frequencies more than high frequencies, because the latter are more sensitive to temporal jitter. Additionally, while the approach alleviates obtaining simply stimulus-evoked coherence, it could also mask phase-locking that is time-locked to the stimulus but not entirely attributable to acoustic temporal features.

From the $180 \mathrm{~s}$ trace of spontaneous activity recorded simultaneously from FAF and AC penetrations, 50 chunks of $3 \mathrm{~s}$ length each were taken. The precise time at which chunks started was chosen randomly in a way that the resulting sub-segments would still be non-overlapping. Each of these paired chunks from FAF and AC were treated as a trial, and iCoh was estimated from all 50 of them for the corresponding penetration (data shown in Fig. 3B). A surrogate calculation was performed whereby the precise phase relationship between FAF and AC "trials" in spontaneous activity was abolished. This was accomplished by pairing FAF chunks with AC chunks randomly. The former affects the timing of the phase-relationships but maintains the overall power across selected chunks. The pairing was performed randomly a sufficiently large number of times (250), and iCoh was calculated at each repetition of the surrogate analysis. Thus, it was possible to obtain a distribution of iCoh values that represented coherence estimates in the absence of consistent phase relationships between $\mathrm{AC}$ and FAF during spontaneous activity. The iCoh calculated from the original data was then related to the surrogate iCoh values by means of z-normalization (z-iCoh). At a population level, lack of consistent phase coherence between FAF and AC at a certain frequency would yield z-iCoh values close to 0 . 
coherogram was constructed by calculating coherence in a sliding window of $200 \mathrm{~ms}$ length, which was advanced in steps of $2 \mathrm{~ms}$. Because of the spectral resolution due to window length, the coherence spectrum for frequencies below $4 \mathrm{~Hz}$ could not be estimated with precision. As with any time-frequency resolved approach, there is a compromise between temporal and spectral resolutions, which we empirically found to be best balanced with a $200 \mathrm{~ms}$ window. All power spectra in the time-resolved analysis were obtained with the multitaper method (Percival and Walden, 1993), available in the Chronux toolbox, using 3 tapers and a time-bandwidth (TW) product 2.

The frequency range of 4-12 Hz was used as a representative of low-frequencies in the spectrum, and the $25-45 \mathrm{~Hz}$ band was considered as low-gamma. For comparing iCoh values during sound presentation vs. iCoh values during spontaneous activity, we calculated time-resolved iCoh during spontaneous activity using the same segments with which non-time resolved coherency was calculated (shown in Fig. 3C). Because the length of the spontaneous segments (3 s) was not precisely equal to the length of the stimuli (the Poisson process, for example, was $4 \mathrm{~s}$ long), we processing in a time-resolved manner as follows:

$$
i \operatorname{Coh}_{\text {increase }}(\omega, t)=\frac{i \operatorname{Coh}_{\text {stim }}(\omega, t)-i \operatorname{Coh}_{\text {spont }}(\omega)}{i \operatorname{Coh}_{\text {spont }}(\omega)} * 100
$$

776

where $i \operatorname{Coh}_{\text {stim }}(\omega, t)$ is the iCoh value during stimulus presentation at frequency $\omega$ ant time $t$, while $i \operatorname{Coh}_{\text {spont }}(\omega)$ is the collapsed time-resolved iCoh during spontaneous activity at the same frequency. The percentage increases were narrowed to the frequency bands of interest (i.e. 4-12 and 25-45 Hz) by calculating the median iCoh increase in the corresponding frequency range over time (data depicted in Fig. 5A, D, G, J). For evaluating iCoh increase at stimulus onset, the median was calculated not only for the frequency range, but also across time in the first $100 \mathrm{~ms}$ after the sequence onset.

\subsection{LFP onset power analyses}

To test to what extent coherent gamma oscillations in the FAF-AC network could be attributable to a broadband evoked response in the LFP, we explored the statistical dependence of gamma power on a trial by trial basis. Spectral properties were obtained using three different temporal windows (Fig. 6): pre $(-110 \mathrm{~ms}--20 \mathrm{~ms}$ relative to stimulus onset), onset ( $0-90 \mathrm{~ms}$ relative to stimulus onset), late 

were performed using the $5.28 \mathrm{~Hz}$ syllabic train, thereby guaranteeing that responses to only one syllable (i.e. the first syllable presented) were considered in the time windows of choice. All spectra were calculated using the Chronux toolbox, with 2 tapers and a TW product of 2, on a trial-by-trial basis. Power spectra were compared for every penetration, per trial, statistically probing changes in the power of low-frequency $(0-15 \mathrm{~Hz})$ and gamma bands $(25-45,45-80$, and $25-80 \mathrm{~Hz})$ in the pre vs. onset windows (Fig. S2A; Wilcoxon signed rank tests, significance when p < 0.01), as well as during the pre vs. late periods (Fig. 6C). A percentage of significant difference (ratio across 50 penetrations) is depicted in the abovementioned figures. The time-frequency analyses shown in Fig. 6D was obtained by evaluating significance differences, per penetration, at given time windows (90 ms length) which were slid (10 ms steps) over times surrounding stimulus onset. Each time window spectra were compared, on a trial-by-trial basis given a penetration, with a window located before stimulus onset (center at ca. $-90 \mathrm{~ms}$ relative to onset; Wilcoxon signed rank tests, significance when $\mathrm{p}$ $<0.01)$.

As per Medvedev and Kanwal (2008), the power spectra from each penetration were z-normalized across trials, and the power in a given band was calculated by integrating (trapz function, Matlab) over the z-normalized spectrum (per trial). Care was taken that the number of frequency samples were comparable when integrating at different bands; the gamma band was therefore divided into 2545 and $45-60 \mathrm{~Hz}$ sub-bands. We then determined whether the distribution of power in gamma and low-frequencies were different, per penetration, by means of a 2-sample Kolmogorov Smirnov test (alpha at 0.01). Because differences or lack of differences in the power distributions do not

809 necessarily imply the existence (or lack) of trial-by-trial correlation between the power of low and

810 gamma frequency bands, we tested whether these powers were correlated for every penetration, on a 811 trial by trial basis. In this context, correlations were significant given a $p<0.01$.

\section{$812 \quad 4.8 \quad$ Information theoretic analyses}

813 Information in the neuronal response regarding the acoustic stimuli was quantified by means of

814 Shannon's mutual information (MI; (Shannon, 2001)). The MI between a stimulus set $S$ and a 815 response set $R$ is mathematically expressed as follows: 
817 where $H(R)$ is the response entropy (i.e. the overall variability of the response set), which is

818 expressed as:

$$
H(R)=-\sum_{r \in R} P(r) \log _{2}[P(r)]
$$

while

$$
H(R \mid S)=-\sum_{s \in S} P(s) \sum_{r \in R} P(r \mid s) \log _{2}[P(r \mid s)], \quad[6]
$$

822 is referred to as the "noise entropy", representing the irreproducibility of the response given a

823 stimulus. The probabilities $P(r), P(s)$ and $P(r \mid s)$ indicate the probability of observing response $r$ taken

824 from the set $R$, the probability of observing stimulus $s$ from the set $S$, and the probability of observing

825 response $r$ given stimulus $s$, respectively. If the logarithm in Eqs. 5 and 6 is of base 2, the MI has

826 units of bits. Each bit of information means that an external observer is able to reduce, by observing

827 the response, the uncertainty about the stimulus by a factor of 2 on a single trial basis. These

828 quantities were estimated by means of the Information Breakdown Toolbox (ibTB; (Magri et al., 829 2009)).

\section{$830 \quad$ 4.8.1 Stimuli for MI computations}

831 With aims of quantifying the amount of information provided by FAF and AC spiking regarding a 832 specific acoustic stream, we calculated each unit's ability to discriminate consecutive chunks of the 833 stimulus from each other (de Ruyter van Steveninck et al., 1997; Kayser et al., 2009; Kayser et al.,

834 2010; Garcia-Rosales et al., 2018a). A particular sequence $S$ (be it, for example, the natural the

835 distress call) was subdivided into non-overlapping, consecutive segments $s_{k}(\mathrm{k}=1,2,3, \ldots, \mathrm{M})$, all of 836 length $\mathrm{T}=4 \mathrm{~ms}$. We chose this segment length so that results from this paper would be comparable 837 with previous data from the AC of C. perspicillata (Garcia-Rosales et al., 2018a). Using lengths in 838 the range of 2-12 ms did not alter the results qualitatively. Each segment $s$ was treated as an 839 independent substimulus from the set $S$. Note that, in this framework, all substimuli are equiprobable.

\section{$840 \quad$ 4.8.2 Rate and joint neuronal codes}

841 The manner in which $P(r)$ is quantified depends directly on the assumptions made to characterize the 842 neuronal response (i.e. the neural code considered). Here we used a rate code $\left(\mathrm{I}_{\text {rate }}\right)$, which determines 843 how well a unit discriminates between each substimulus $s$, based on its spiking rate. The response set 844 represented whether a spike occurred or not, and can be characterized as follows: $R=\{0,1\}$, where 1 845 and 0 represent the occurrence or absence of a spike, respectively. $P(r)$ was then the probability that a 
unit fired or not a spike across all trials, whereas $P(r \mid s)$ was the probability of firing to a certain substimulus. The time window was sufficiently short to assume that, in general, a single spike would occur within each time segment, and therefore binarized responses were used for MI calculations.

849 The information provided by joint responses from the FAF and the AC ( $\left.\mathrm{I}_{\text {joint }}\right)$ was calculated by taking into account which unit elicited a spike in a merged response (see Fig. 6B; also referred to as "line code" in the literature (Panzeri et al., 2007; Kayser et al., 2009)). That is, the response set was defined as $R=\{(0,0) ;(0,1) ;(1,0) ;(1,1)\}$, where each member of the set represents whether and which unit fired a spike (e.g. $(0,1)$ could indicate that the FAF unit did not fire, whereas the AC one did; $(1,0)$ would represent the opposite).

\subsubsection{Quantifying information from limited samples}

The probabilities in Eqs. 5 and $\mathbf{6}$ are estimated empirically from the data, based on the representation of neuronal responses described above (i.e. the neural codes). These empirically estimated probabilities (such as $P(r)$, or $P(r \mid s)$ ) are biased because it is impossible in practice to sample all possible values of $R$ a sufficiently large number of times (ideally, infinite). A number of methods have been developed to deal with the sampling bias (Panzeri et al., 2007). In this study, we used the Quadratic Extrapolation (QE) procedure (Strong et al., 1998), implemented in the ibTB. In addition to the $\mathrm{QE}$, we subtracted possible remaining biases by means of a bootstrap procedure (Montemurro et al., 2008; Garcia-Rosales et al., 2018a), using 250 repetitions. For paired responses, we also used the shuffling procedure (Panzeri et al., 2007) implemented in the iBTB together with the bootstrap method. To corroborate that the information estimates presented in the results were not affected by the limited sampling bias, we conducted numerical simulations in order to measure the dependence of the bias on the number of trials. The results of these simulations are shown in Fig. S8, and indicate that the number of trials used in this study (50) was sufficient to robustly estimate the information quantities presented.

\subsection{Statistical analyses}

871 All statistical analyses were conducted in Matlab (version 8.6.0.267246 (R2015b)), with custom872 written scripts using the Statistics and Machine Learning Toolbox. Tests for comparisons between 873 the distributions of the quantities described above were always indicated in the main text. When 874 multiple comparisons were done, we performed False-Discovery Rate (FDR) corrections (e.g., 875 comparing across multiple channel pairs in Fig. 3E) with the Benjamini and Hochberg procedure 
876 (Benjamini and Hochberg, 1995). The significance threshold was set at an alpha of 0.05. If the $\mathrm{p}$

877 values reported were uncorrected, it is stated so in the text. Effect sizes were calculated with the $r$

878 metric, which is defined as follows (Fritz et al., 2012):

879

$$
r=\frac{W}{\sqrt{N}}
$$

880 where $r$ is the effect size, $W$ is the test statistic of the Wilcoxon signed rank test used in this context,

881 and $\mathrm{N}$ is the sample size of the quantities being compared $(\mathrm{N}=50)$. Values of $r \leq 0.3$ were

882 considered small effects, while $0.3 \leq r \leq 0.5$ were considered as medium effects, and large effects

883 were considered when $\mathrm{r}>0.5$ (Fritz et al., 2012).

8845 Conflict of Interest

885 The authors declare that the research was conducted in the absence of any commercial or financial 886 relationships that could be construed as a potential conflict of interest.

\section{Author Contributions}

888 F.G.R and J.C.H designed the study. F.G.R collected the data. F.G.R analyzed the data and wrote 889 the manuscript. F.G.R, L.L.J, E.G.P, Y.C.C, and J.C.H discussed the results and reviewed the 890 manuscript.

\section{$891 \quad 7 \quad$ Acknowledgments}

892 We are thankful to Manfred Kössl for comments on an earlier version of this manuscript.

\section{$893 \quad 8 \quad$ Funding}

894 The German Research Council (DFG) founded this work (Grant No. HE 7478/1-1, to JCH).

\section{References}

896 Arnal, L.H., and Giraud, A.L. (2012). Cortical oscillations and sensory predictions. Trends Cogn Sci 897 16, 390-398.

898 Bartenstein, S.K., Gerstenberg, N., Vanderelst, D., Peremans, H., and Firzlaff, U. (2014). Echo-

899 acoustic flow dynamically modifies the cortical map of target range in bats. Nature Communications $900 \quad 5$. 
901 Bartoli, E., Bosking, W., Chen, Y., Li, Y., Sheth, S.A., Beauchamp, M.S., Yoshor, D., and Foster, 902 B.L. (2019). Functionally Distinct Gamma Range Activity Revealed by Stimulus Tuning in Human 903 Visual Cortex. Curr Biol 29, 3345-3358 e3347.

904 Bastos, A.M., and Schoffelen, J.M. (2015). A Tutorial Review of Functional Connectivity Analysis 905 Methods and Their Interpretational Pitfalls. Front Syst Neurosci 9, 175.

906 Beetz, M.J., Hechavarria, J.C., and Kossl, M. (2016). Cortical neurons of bats respond best to echoes 907 from nearest targets when listening to natural biosonar multi-echo streams. Sci Rep 6, 35991.

908 Benjamini, Y., and Hochberg, Y. (1995). Controlling the False Discovery Rate - a Practical and 909 Powerful Approach to Multiple Testing. J Roy Stat Soc B Met 57, 289-300.

910 Bokil, H., Andrews, P., Kulkarni, J.E., Mehta, S., and Mitra, P.P. (2010). Chronux: a platform for 911 analyzing neural signals. Journal of neuroscience methods 192, 146-151.

912 Brosch, M., Budinger, E., and Scheich, H. (2002). Stimulus-related gamma oscillations in primate 913 auditory cortex. J Neurophysiol 87, 2715-2725.

914 Buzsaki, G., Anastassiou, C.A., and Koch, C. (2012). The origin of extracellular fields and currents-915 EEG, ECoG, LFP and spikes. Nat Rev Neurosci 13, 407-420.

916 Cambiaghi, M., Grosso, A., Likhtik, E., Mazziotti, R., Concina, G., Renna, A., Sacco, T., Gordon, 917 J.A., and Sacchetti, B. (2016). Higher-Order Sensory Cortex Drives Basolateral Amygdala Activity 918 during the Recall of Remote, but Not Recently Learned Fearful Memories. J Neurosci 36, 1647-1659.

919 Carbajal, G.V., and Malmierca, M.S. (2018). The Neuronal Basis of Predictive Coding Along the 920 Auditory Pathway: From the Subcortical Roots to Cortical Deviance Detection. Trends Hear 22, 9212331216518784822.

922 Casseday, J.H., Kobler, J.B., Isbey, S.F., and Covey, E. (1989). Central acoustic tract in an

923 echolocating bat: an extralemniscal auditory pathway to the thalamus. J Comp Neurol 287, $247-259$.

924 Chase, S.M., and Young, E.D. (2007). First-spike latency information in single neurons increases 925 when referenced to population onset. Proc Natl Acad Sci U S A 104, 5175-5180.

926 Cho, R.Y., Konecky, R.O., and Carter, C.S. (2006). Impairments in frontal cortical gamma

927 synchrony and cognitive control in schizophrenia. Proc Natl Acad Sci U S A 103, 19878-19883.

928 Concina, G., Renna, A., Grosso, A., and Sacchetti, B. (2019). The auditory cortex and the emotional 929 valence of sounds. Neurosci Biobehav R 98, 256-264.

930 Curic, S., Leicht, G., Thiebes, S., Andreou, C., Polomac, N., Eichler, I.C., Eichler, L., Zollner, C., 931 Gallinat, J., Steinmann, S., et al. (2019). Reduced auditory evoked gamma-band response and 932 schizophrenia-like clinical symptoms under subanesthetic ketamine. Neuropsychopharmacology 44, 933 1239-1246.

934 Daume, J., Gruber, T., Engel, A.K., and Friese, U. (2017). Phase-Amplitude Coupling and Long935 Range Phase Synchronization Reveal Frontotemporal Interactions during Visual Working Memory. J 936 Neurosci 37, 313-322.

937 de Ruyter van Steveninck, R.R., Lewen, G.D., Strong, S.P., Koberle, R., and Bialek, W. (1997).

938 Reproducibility and variability in neural spike trains. Science 275, 1805-1808.

939 Debener, S., Herrmann, C.S., Kranczioch, C., Gembris, D., and Engel, A.K. (2003). Top-down 940 attentional processing enhances auditory evoked gamma band activity. Neuroreport 14, 683-686. 
941 Durschmid, S., Edwards, E., Reichert, C., Dewar, C., Hinrichs, H., Heinze, H.J., Kirsch, H.E., Dalal,

942 S.S., Deouell, L.Y., and Knight, R.T. (2016). Hierarchy of prediction errors for auditory events in

943 human temporal and frontal cortex. P Natl Acad Sci USA 113, 6755-6760.

944 Durschmid, S., Reichert, C., Hinrichs, H., Heinze, H.J., Kirsch, H.E., Knight, R.T., and Deouell, L.Y. 945 (2018). Direct Evidence for Prediction Signals in Frontal Cortex Independent of Prediction Error.

946 Cereb Cortex.

947 Eiermann, A., and Esser, K.H. (2000). Auditory responses from the frontal cortex in the short-tailed

948 fruit bat Carollia perspicillata. Neuroreport 11, 421-425.

949 Esser, K.H., and Eiermann, A. (1999). Tonotopic organization and parcellation of auditory cortex in 950 the FM-bat Carollia perspicillata. Eur J Neurosci 11, 3669-3682.

951 Floresco, S.B., and Ghods-Sharifi, S. (2007). Amygdala-prefrontal cortical circuitry regulates effort952 based decision making. Cerebral Cortex 17, 251-260.

953 Francis, N.A., Elgueda, D., Englitz, B., Fritz, J.B., and Shamma, S.A. (2018). Laminar profile of

954 task-related plasticity in ferret primary auditory cortex. Scientific Reports 8.

955 Fries, P. (2009). Neuronal gamma-band synchronization as a fundamental process in cortical

956 computation. Annu Rev Neurosci 32, 209-224.

957 Fries, P. (2015). Rhythms for Cognition: Communication through Coherence. Neuron 88, 220-235.

958 Friston, K. (2018). Does predictive coding have a future? Nat Neurosci 21, 1019-+.

959 Fritz, C.O., Morris, P.E., and Richler, J.J. (2012). Effect Size Estimates: Current Use, Calculations, 960 and Interpretation. J Exp Psychol Gen 141, 2-18.

961 Fuster, J.M., Bodner, M., and Kroger, J.K. (2000). Cross-modal and cross-temporal association in

962 neurons of frontal cortex. Nature 405, 347-351.

963 Gaese, B.H., and Ostwald, J. (1995). Temporal coding of amplitude and frequency modulation in the 964 rat auditory cortex. Eur J Neurosci 7, 438-450.

965 Gao, X., and Wehr, M. (2015). A coding transformation for temporally structured sounds within 966 auditory cortical neurons. Neuron 86, 292-303.

967 Garcia-Rosales, F., Beetz, M.J., Cabral-Calderin, Y., Kossl, M., and Hechavarria, J.C. (2018a).

968 Neuronal coding of multiscale temporal features in communication sequences within the bat auditory 969 cortex. Commun Biol 1, 200.

970 Garcia-Rosales, F., Martin, L.M., Beetz, M.J., Cabral-Calderin, Y., Kossl, M., and Hechavarria, J.C. 971 (2018b). Low-Frequency Spike-Field Coherence Is a Fingerprint of Periodicity Coding in the 972 Auditory Cortex. iScience 9, 47-62.

973 Garcia-Rosales, F., Rohrig, D., Weineck, K., Rohm, M., Lin, Y.H., Cabral-Calderin, Y., Kossl, M., 974 and Hechavarria, J.C. (2019). Laminar specificity of oscillatory coherence in the auditory cortex.

975 Brain Struct Funct 224, 2907-2924.

976 Gaucher, Q., Huetz, C., Gourevitch, B., Laudanski, J., Occelli, F., and Edeline, J.M. (2013). How do 977 auditory cortex neurons represent communication sounds? Hear Res 305, 102-112.

978 Gilmartin, M.R., Balderston, N.L., and Helmstetter, F.J. (2014). Prefrontal cortical regulation of fear 979 learning. Trends Neurosci 37, 455-464.

980 Giraud, A.L., and Poeppel, D. (2012). Cortical oscillations and speech processing: emerging 981 computational principles and operations. Nat Neurosci 15, 511-517. 
982 Gourley, S.L., Olevska, A., Zimmermann, K.S., Ressler, K.J., Dileone, R.J., and Taylor, J.R. (2013).

983 The orbitofrontal cortex regulates outcome-based decision-making via the lateral striatum. Eur J

984 Neurosci 38, 2382-2388.

985 Gurtubay, I.G., Alegre, M., Labarga, A., Malanda, A., and Artieda, J. (2004). Gamma band responses 986 to target and non-target auditory stimuli in humans. Neurosci Lett 367, 6-9.

987 Hage, S.R., and Nieder, A. (2015). Audio-vocal interaction in single neurons of the monkey

988 ventrolateral prefrontal cortex. J Neurosci 35, 7030-7040.

989 Hechavarria, J.C., Beetz, M.J., Macias, S., and Kossl, M. (2016a). Distress vocalization sequences

990 broadcasted by bats carry redundant information. J Comp Physiol A Neuroethol Sens Neural Behav

991 Physiol 202, 503-515.

992 Hechavarria, J.C., Beetz, M.J., Macias, S., and Kossl, M. (2016b). Vocal sequences suppress spiking

993 in the bat auditory cortex while evoking concomitant steady-state local field potentials. Sci Rep 6,

99439226.

995 Hechavarria, J.C., Macias, S., Vater, M., Voss, C., Mora, E.C., and Kossl, M. (2013). Blurry

996 topography for precise target-distance computations in the auditory cortex of echolocating bats. Nat

997 Commun 4, 2587.

998 Helfrich, R.F., Huang, M., Wilson, G., and Knight, R.T. (2017). Prefrontal cortex modulates

999 posterior alpha oscillations during top-down guided visual perception. Proc Natl Acad Sci U S A

$1000114,9457-9462$.

1001 Helfrich, R.F., and Knight, R.T. (2016). Oscillatory Dynamics of Prefrontal Cognitive Control.

1002 Trends Cogn Sci 20, 916-930.

1003 Henrie, J.A., and Shapley, R. (2005). LFP power spectra in V1 cortex: the graded effect of stimulus

1004 contrast. J Neurophysiol 94, 479-490.

1005 Hermes, D., Miller, K.J., Wandell, B.A., and Winawer, J. (2015). Stimulus Dependence of Gamma

1006 Oscillations in Human Visual Cortex. Cereb Cortex 25, 2951-2959.

1007 Hwang, J., and Romanski, L.M. (2015). Prefrontal neuronal responses during audiovisual mnemonic 1008 processing. J Neurosci 35, 960-971.

1009 Hyafil, A., Fontolan, L., Kabdebon, C., Gutkin, B., and Giraud, A.L. (2015). Speech encoding by

1010 coupled cortical theta and gamma oscillations. Elife 4, e06213.

1011 Imada, A., Morris, A., and Wiest, M.C. (2012). Deviance detection by a P3-like response in rat

1012 posterior parietal cortex. Front Integr Neurosci 6, 127.

1013 Insanally, M.N., Carcea, I., Field, R.E., Rodgers, C.C., DePasquale, B., Rajan, K., DeWeese, M.R.,

1014 Albanna, B.F., and Froemke, R.C. (2019). Spike-timing-dependent ensemble encoding by non-

1015 classically responsive cortical neurons. Elife 8.

1016 Kanold, P.O., Nelken, I., and Polley, D.B. (2014). Local versus global scales of organization in 1017 auditory cortex. Trends Neurosci 37, 502-510.

1018 Kanwal, J.S., Gordon, M., Peng, J.P., and Heinz-Esser, K. (2000). Auditory responses from the 1019 frontal cortex in the mustached bat, Pteronotus parnellii. Neuroreport 11, 367-372.

1020 Karalis, N., Dejean, C., Chaudun, F., Khoder, S., Rozeske, R.R., Wurtz, H., Bagur, S., Benchenane, 1021 K., Sirota, A., Courtin, J., et al. (2016). 4-Hz oscillations synchronize prefrontal-amygdala circuits

1022 during fear behavior. Nat Neurosci 19, 605-612. 
1023 Kayser, C., Ince, R.A., and Panzeri, S. (2012). Analysis of slow (theta) oscillations as a potential

1024 temporal reference frame for information coding in sensory cortices. PLoS Comput Biol 8,

$1025 \mathrm{e} 1002717$.

1026 Kayser, C., Logothetis, N.K., and Panzeri, S. (2010). Millisecond encoding precision of auditory 1027 cortex neurons. Proc Natl Acad Sci U S A 107, 16976-16981.

1028 Kayser, C., Montemurro, M.A., Logothetis, N.K., and Panzeri, S. (2009). Spike-phase coding boosts 1029 and stabilizes information carried by spatial and temporal spike patterns. Neuron 61, 597-608.

1030 Kikuchi, Y., Attaheri, A., Wilson, B., Rhone, A.E., Nourski, K.V., Gander, P.E., Kovach, C.K., 1031 Kawasaki, H., Griffiths, T.D., Howard, M.A., et al. (2017). Sequence learning modulates neural 1032 responses and oscillatory coupling in human and monkey auditory cortex. Plos Biology 15.

1033 Kim, H., Ahrlund-Richter, S., Wang, X., Deisseroth, K., and Carlen, M. (2016). Prefrontal

1034 Parvalbumin Neurons in Control of Attention. Cell 164, 208-218.

1035 Kobler, J.B., Isbey, S.F., and Casseday, J.H. (1987). Auditory Pathways to the Frontal-Cortex of the 1036 Moustache Bat, Pteronotus-Parnellii. Science 236, 824-826.

1037 Lakatos, P., Shah, A.S., Knuth, K.H., Ulbert, I., Karmos, G., and Schroeder, C.E. (2005). An oscillatory hierarchy controlling neuronal excitability and stimulus processing in the auditory cortex. J Neurophysiol 94, 1904-1911.

1040 Leicht, G., Kirsch, V., Giegling, I., Karch, S., Hantschk, I., Moller, H.J., Pogarell, O., Hegerl, U., Rujescu, D., and Mulert, C. (2010). Reduced early auditory evoked gamma-band response in patients 1042 with schizophrenia. Biol Psychiatry 67, 224-231.

1043 Levakova, M., Tamborrino, M., Ditlevsen, S., and Lansky, P. (2015). A review of the methods for 1044 neuronal response latency estimation. Biosystems 136, 23-34.

1045 Li, J.C., Liao, X., Zhang, J.X., Wang, M., Yang, N., Zhang, J., Lv, G.H., Li, H.H., Lu, J., Ding, R., et 1046 al. (2017). Primary Auditory Cortex is Required for Anticipatory Motor Response. Cerebral Cortex 1047 27, 3254-3271.

1048 Lopez-Jury, L., Mannel, A., Garcia-Rosales, F., and Hechavarria, J.C. (2019). Modified synaptic dynamics predict neural activity patterns in an auditory field within the frontal cortex. Eur J

1051 Lu, Q., Jiang, C., and Zhang, J. (2016). Encoding of sound envelope transients in the auditory cortex 1052 of juvenile rats and adult rats. Int J Dev Neurosci 48, 50-57.

1053 Lu, T., Liang, L., and Wang, X. (2001). Temporal and rate representations of time-varying signals in 1054 the auditory cortex of awake primates. Nat Neurosci 4, 1131-1138.

1055 Magri, C., Whittingstall, K., Singh, V., Logothetis, N.K., and Panzeri, S. (2009). A toolbox for the 1056 fast information analysis of multiple-site LFP, EEG and spike train recordings. BMC Neurosci 10, 105781.

1058 Martin, L.M., Garcia-Rosales, F., Beetz, M.J., and Hechavarria, J.C. (2017). Processing of 1059 temporally patterned sounds in the auditory cortex of Seba's short-tailed bat,Carollia perspicillata.

1060 Eur J Neurosci 46, 2365-2379.

1061 Masquelier, T. (2013). Neural variability, or lack thereof. Front Comput Neurosci 7, 7.

1062 Medalla, M., and Barbas, H. (2014). Specialized prefrontal "auditory fields": organization of primate 1063 prefrontal-temporal pathways. Front Neurosci 8, 77. 
1064 Medvedev, A.V., and Kanwal, J.S. (2008). Communication call-evoked gamma-band activity in the 1065 auditory cortex of awake bats is modified by complex acoustic features. Brain Res 1188, 76-86.

1066 Miller, E.K. (2000). The prefrontal cortex and cognitive control. Nat Rev Neurosci 1, 59-65.

1067 Montemurro, M.A., Rasch, M.J., Murayama, Y., Logothetis, N.K., and Panzeri, S. (2008). Phase-of1068 firing coding of natural visual stimuli in primary visual cortex. Curr Biol 18, 375-380.

1069 Mrsic-Flogel, T.D., King, A.J., and Schnupp, J.W. (2005). Encoding of virtual acoustic space stimuli 1070 by neurons in ferret primary auditory cortex. J Neurophysiol 93, 3489-3503.

1071 Mulert, C., Leicht, G., Pogarell, O., Mergl, R., Karch, S., Juckel, G., Moller, H.J., and Hegerl, U. 1072 (2007). Auditory cortex and anterior cingulate cortex sources of the early evoked gamma-band 1073 response: relationship to task difficulty and mental effort. Neuropsychologia 45, 2294-2306.

1074 Murty, D., Shirhatti, V., Ravishankar, P., and Ray, S. (2018). Large Visual Stimuli Induce Two 1075 Distinct Gamma Oscillations in Primate Visual Cortex. J Neurosci 38, 2730-2744.

1076 Newman, J.D., and Lindsley, D.F. (1976). Single Unit Analysis of Auditory Processing in Squirrel1077 Monkey Frontal Cortex. Experimental Brain Research 25, 169-181.

1078 Nolte, G., Bai, O., Wheaton, L., Mari, Z., Vorbach, S., and Hallett, M. (2004). Identifying true brain 1079 interaction from EEG data using the imaginary part of coherency. Clin Neurophysiol 115, 2292-

10802307.

1081 Panzeri, S., Senatore, R., Montemurro, M.A., and Petersen, R.S. (2007). Correcting for the sampling 1082 bias problem in spike train information measures. J Neurophysiol 98, 1064-1072.

1083 Park, H., Ince, R.A., Schyns, P.G., Thut, G., and Gross, J. (2015). Frontal top-down signals increase 1084 coupling of auditory low-frequency oscillations to continuous speech in human listeners. Curr Biol $108525,1649-1653$.

1086 Parras, G.G., Nieto-Diego, J., Carbajal, G.V., Valdes-Baizabal, C., Escera, C., and Malmierca, M.S. 1087 (2017). Neurons along the auditory pathway exhibit a hierarchical organization of prediction error.

1088 Nat Commun 8, 2148.

1089 Percival, D.B., and Walden, A.T. (1993). Spectral analysis for physical applications (cambridge 1090 university press).

1091 Pezze, M., McGarrity, S., Mason, R., Fone, K.C., and Bast, T. (2014). Too little and too much:

1092 hypoactivation and disinhibition of medial prefrontal cortex cause attentional deficits. J Neurosci 34, 1093 7931-7946.

1094 Plakke, B., and Romanski, L.M. (2014). Auditory connections and functions of prefrontal cortex.

1095 Front Neurosci-Switz 8.

1096 Polomac, N., Leicht, G., Nolte, G., Andreou, C., Schneider, T.R., Steinmann, S., Engel, A.K., and 1097 Mulert, C. (2015). Generators and Connectivity of the Early Auditory Evoked Gamma Band 1098 Response. Brain Topogr 28, 865-878.

1099 Popa, D., Duvarci, S., Popescu, A.T., Lena, C., and Pare, D. (2010). Coherent amygdalocortical theta 1100 promotes fear memory consolidation during paradoxical sleep. Proc Natl Acad Sci U S A 107, 651611016519.

1102 Ray, S., and Maunsell, J.H. (2010). Differences in gamma frequencies across visual cortex restrict 1103 their possible use in computation. Neuron 67, 885-896. 
1104 Recanzone, G.H. (2000). Spatial processing in the auditory cortex of the macaque monkey. Proc Natl 1105 Acad Sci U S A 97, 11829-11835.

1106 Risterucci, C., Terramorsi, D., Nieoullon, A., and Amalric, M. (2003). Excitotoxic lesions of the 1107 prelimbic-infralimbic areas of the rodent prefrontal cortex disrupt motor preparatory processes. Eur $\mathbf{J}$ 1108 Neurosci 17, 1498-1508.

1109 Romanski, L.M. (2007). Representation and integration of auditory and visual stimuli in the primate 1110 ventral lateral prefrontal cortex. Cereb Cortex 17 Suppl 1, i61-69.

1111 Romanski, L.M., and Goldman-Rakic, P.S. (2002). An auditory domain in primate prefrontal cortex. 1112 Nat Neurosci 5, 15-16.

1113 Runyan, C.A., Piasini, E., Panzeri, S., and Harvey, C.D. (2017). Distinct timescales of population 1114 coding across cortex. Nature 548, 92-96.

1115 Sakata, S., and Harris, K.D. (2009). Laminar structure of spontaneous and sensory-evoked population 1116 activity in auditory cortex. Neuron 64, 404-418.

1117 Salminen, N.H., Takanen, M., Santala, O., Lamminsalo, J., Altoe, A., and Pulkki, V. (2015).

1118 Integrated processing of spatial cues in human auditory cortex. Hear Res 327, 143-152.

1119 Shannon, C.E. (2001). A mathematical theory of communication. ACM SIGMOBILE Mobile 1120 Computing and Communications Review 5, 3-55.

1121 Sheikh, A.S., Harper, N.S., Drefs, J., Singer, Y., Dai, Z., Turner, R.E., and Lucke, J. (2019). STRFs 1122 in primary auditory cortex emerge from masking-based statistics of natural sounds. PLoS Comput 1123 Biol 15, e1006595.

1124 Song, E.Y., Boatman, J.A., Jung, M.W., and Kim, J.J. (2010). Auditory cortex is important in the 1125 extinction of two different tone-based conditioned fear memories in rats. Front Behav Neurosci 4.

1126 St Onge, J.R., Abhari, H., and Floresco, S.B. (2011). Dissociable Contributions by Prefrontal D-1 1127 and D-2 Receptors to Risk-Based Decision Making. J Neurosci 31, 8625-8633.

1128 Strong, S.P., de Ruyter van Steveninck, R.R., Bialek, W., and Koberle, R. (1998). On the application 1129 of information theory to neural spike trains. Pac Symp Biocomput, 621-632.

1130 Suga, N., and O'Neill, W.E. (1979). Neural axis representing target range in the auditory cortex of the 1131 mustache bat. Science 206, 351-353.

1132 Teng, X., Tian, X., Rowland, J., and Poeppel, D. (2017). Concurrent temporal channels for auditory 1133 processing: Oscillatory neural entrainment reveals segregation of function at different scales. PLoS 1134 Biol 15, e2000812.

1135 Trapeau, R., and Schonwiesner, M. (2018). The Encoding of Sound Source Elevation in the Human 1136 Auditory Cortex. J Neurosci 38, 3252-3264.

1137 Vianney-Rodrigues, P., Iancu, O.D., and Welsh, J.P. (2011). Gamma oscillations in the auditory 1138 cortex of awake rats. Eur J Neurosci 33, 119-129.

1139 Werchan, D.M., Collins, A.G., Frank, M.J., and Amso, D. (2016). Role of Prefrontal Cortex in 1140 Learning and Generalizing Hierarchical Rules in 8-Month-Old Infants. J Neurosci 36, 10314-10322.

1141 Winkowski, D.E., Nagode, D.A., Donaldson, K.J., Yin, P., Shamma, S.A., Fritz, J.B., and Kanold, 1142 P.O. (2018). Orbitofrontal Cortex Neurons Respond to Sound and Activate Primary Auditory Cortex 1143 Neurons. Cereb Cortex 28, 868-879. 
1144 Womelsdorf, T., and Everling, S. (2015). Long-Range Attention Networks: Circuit Motifs

1145 Underlying Endogenously Controlled Stimulus Selection. Trends Neurosci 38, 682-700.

1146 Yger, P., Spampinato, G.L.B., Esposito, E., Lefebvre, B., Deny, S., Gardella, C., Stimberg, M., Jetter, 1147 F., Zeck, G., Picaud, S., et al. (2018). A spike sorting toolbox for up to thousands of electrodes

1148 validated with ground truth recordings in vitro and in vivo. Elife 7.

1149 Yin, P.B., Johnson, J.S., O'Connor, K.N., and Sutter, M.L. (2011). Coding of Amplitude Modulation

1150 in Primary Auditory Cortex. Journal of Neurophysiology 105, 582-600.

1151

\section{Data Availability Statement}

1153 The data that support the findings of this study are available from the corresponding authors upon

1154 reasonable request. 
A

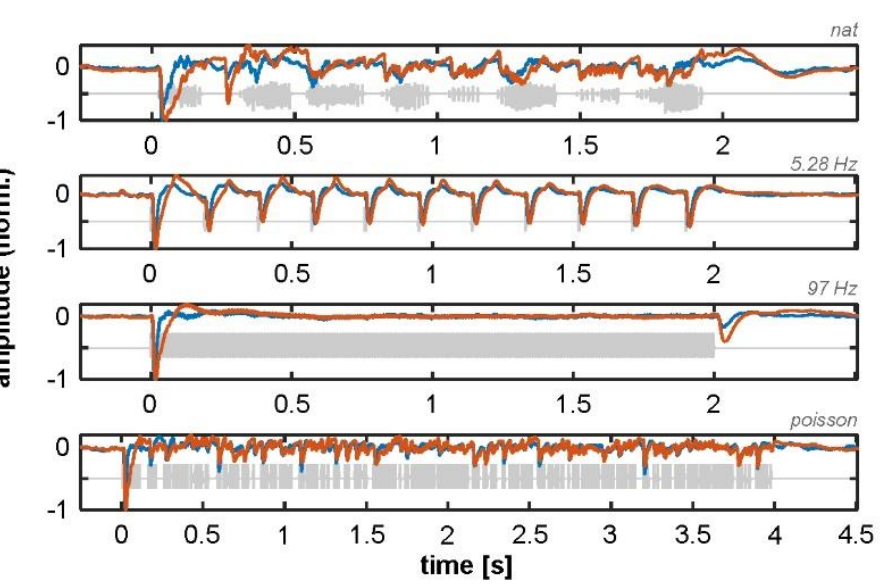

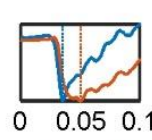

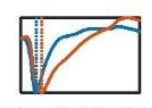

$\begin{array}{llll}0 & 0.05 & 0.1\end{array}$

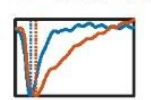

$\begin{array}{lll}0 & 0.05 & 0.1\end{array}$

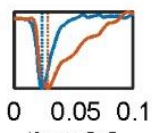

time [s]

C
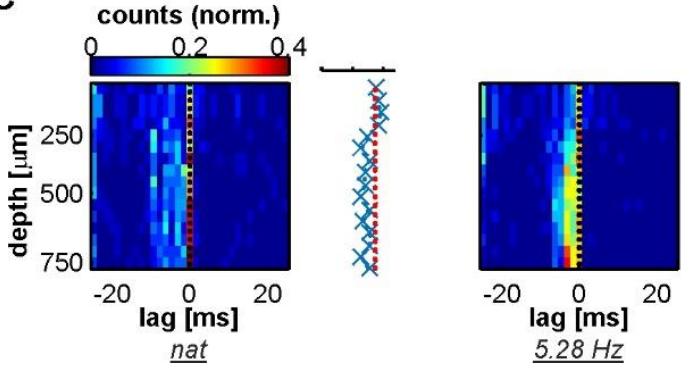

B
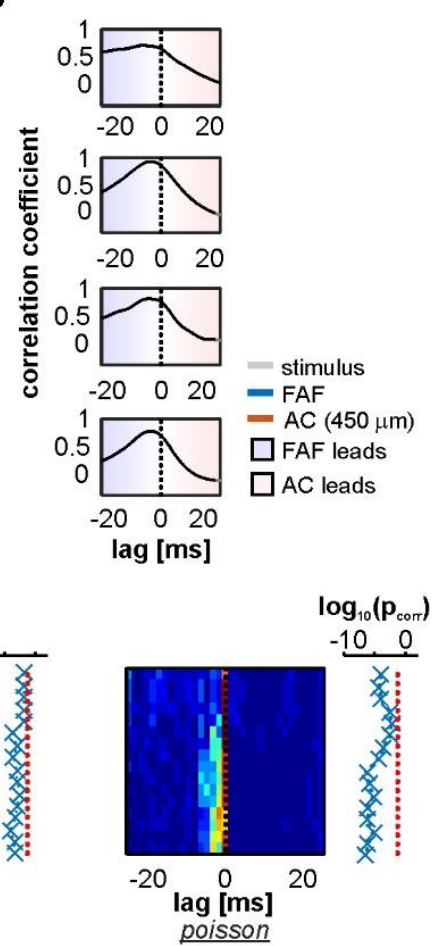

Fig. 1. LFP stimulus-related activity in the frontal auditory field (FAF) precedes that of the recorded from the FAF (blue) and the $\mathrm{AC}$ at a depth of $450 \mu \mathrm{m}$ (orange) in response to the four stimuli tested (ordered from top to bottom: natural sequence (nat), $5.28 \mathrm{~Hz}$ train, $97 \mathrm{~Hz}$ train, and the Poisson syllabic sequence (poisson); grey traces). Right column: zoom into the first $100 \mathrm{~ms}$ after stimulus onset. Negative peaks in the evoked potential are marked with vertical dashed lines. Note that peaks in the FAF occur earlier than in the AC. (B) Cross-correlation between traces in A (left column). Peaks in negative lags indicate that FAF field-potentials lead those in the AC. (C) Peak lags between the cross-correlation of FAF LFPs and AC LFPs, for all penetrations $(n=50)$ and across recording depths. Next to each heatmap, log-scaled corrected $p$ values testing that the peak distribution is significantly below 0 (FDR corrected tailed Wilcoxon signed rank tests; $\mathrm{p}_{\text {corr }}<0.05$ for significance). 
A
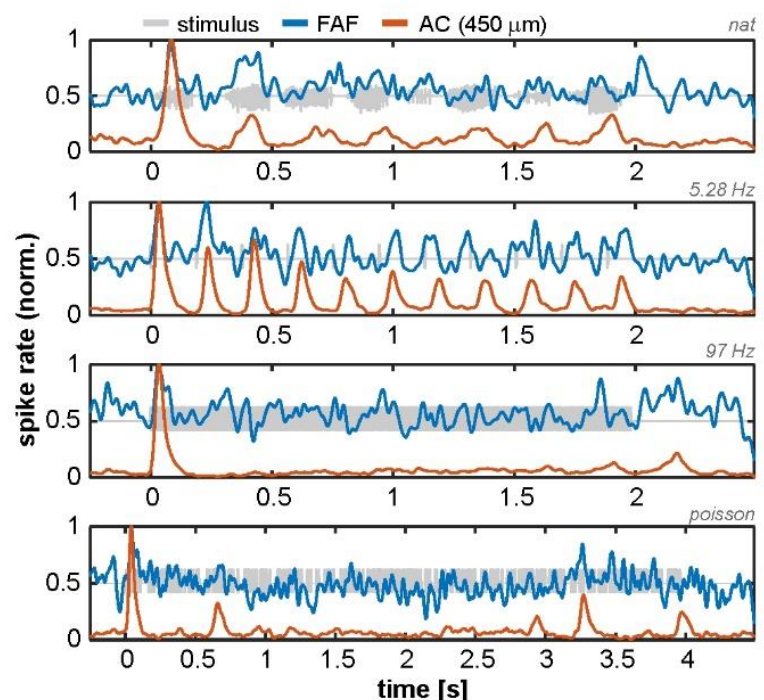

\section{B}

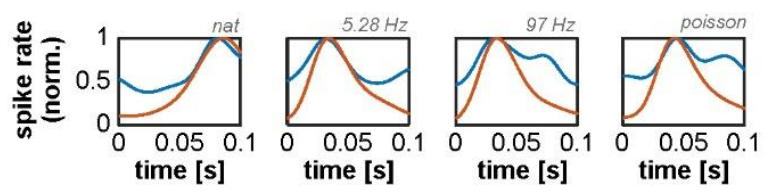

C
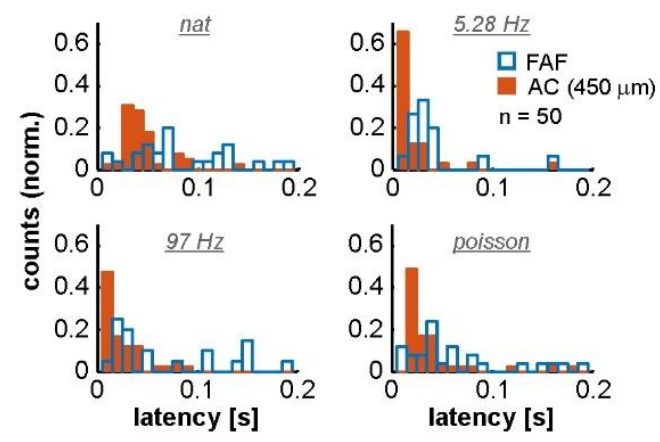

D

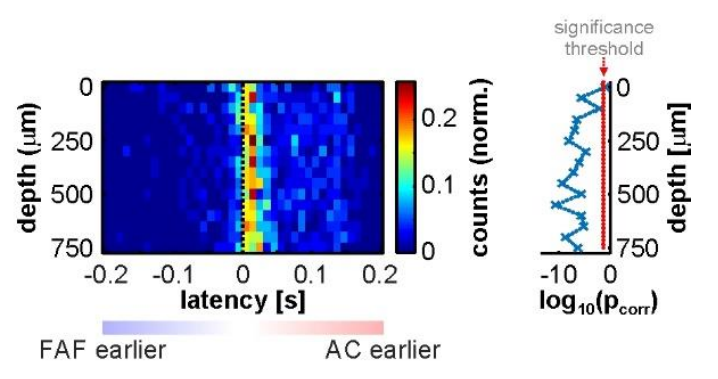

1171 Fig. 2. Spiking activity suggests the presence of fast inputs into the FAF. (A) Spiking responses

1172 from two simultaneously recorded units in the FAF (blue) and the AC (at $450 \mu \mathrm{m}$; blue), in

1173 response to all stimuli tested (top to bottom). (B) Zoom-in into the first $100 \mathrm{~ms}$ after stimulus

1174 onset of the examples shown in A. Note that, for this pair, the peak response for the FAF unit

1175 was at least as fast as the for auditory cortical one. (C) Latency distribution of FAF (blue) units and $\mathrm{AC}$ units at $450 \mu \mathrm{m}$, for all stimuli ( $\mathrm{n}=50$ penetrations). The FAF was, overall, sluggish in comparison to the AC. (D) Response latency difference between simultaneously recorded spiking for FAF and AC at different depths (positive difference, FAF slower than AC; negative difference indicates the opposite). In some cases, FAF spiking responses occurred earlier than $\mathrm{AC}$ responses, although the AC was in general significantly faster than the FAF across channels, except in the case of the most superficial contact (FDR-corrected Wilcoxon signed rank tests, significance when $\left.\mathrm{p}_{\text {corr }}<0.05\right)$. Log-converted $\mathrm{p}$ values and significance threshold are shown to the right of the latency distributions. The threshold is indicated as a red dashed line. 
A

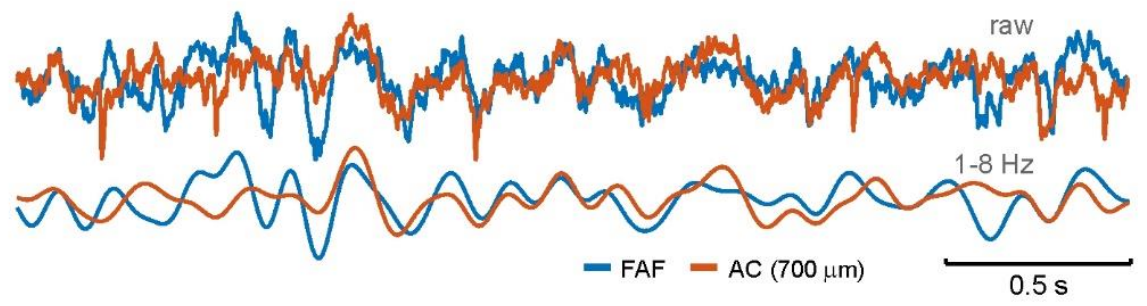

B

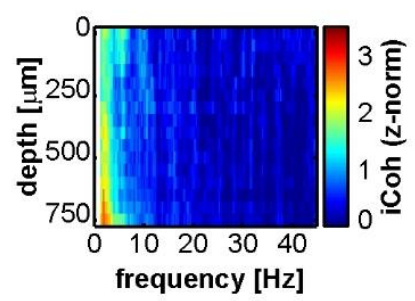

D

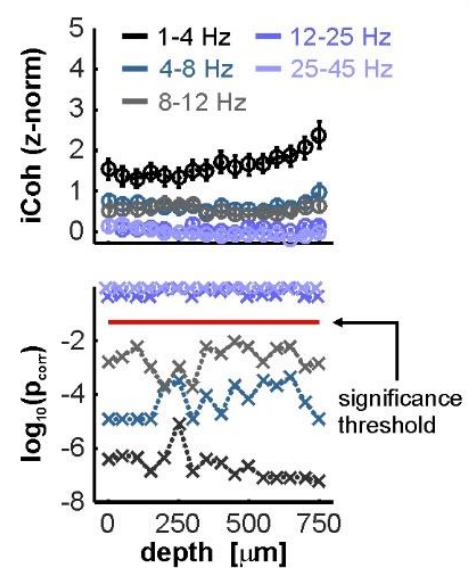

C
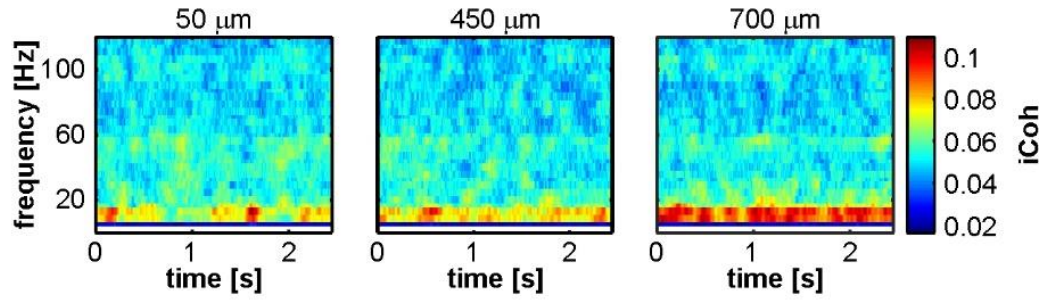

$\mathrm{E}$
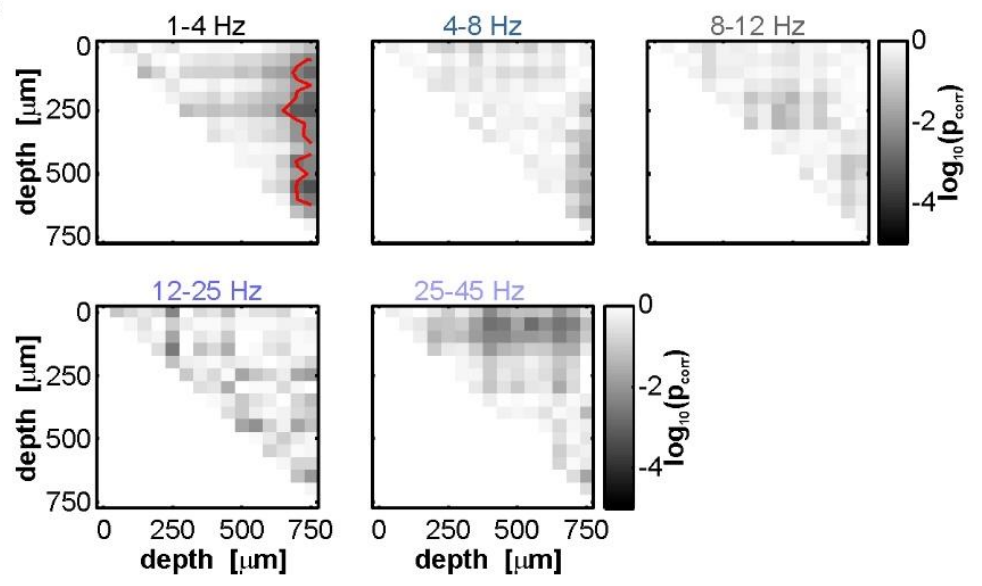

Fig. 3. LFPs from FAF and AC are low-frequency coherent during spontaneous activity in a $\mathrm{AC}$ at a depth of $700 \mu \mathrm{m}$ (orange). Raw (top pair) and low-frequency filtered (bottom pair) traces are shown. (B) Frequency-dependent average imaginary coherence (iCoh), z-normalized to a surrogate distribution, across recording depths in the AC. Deep channels showed, on average, the strongest coherence values at low frequencies. (C) Time-resolved iCoh using the same segments as in B, with a sliding window of $200 \mathrm{~ms}$ (see Methods), the same used for analyzing stimulus-related synchronization. (D) Top: depth-dependent population z-normalized iCoh from B, for distinct frequency bands (delta, theta, alpha, beta and low gamma, see Methods; frequency ranges indicated in the plot), across all penetrations (shown as mean \pm SEM). Bottom: log-scaled corrected p values after testing, per frequency band, whether znormalized iCoh values were significantly higher than 0 across penetrations, per AC depth (FDR-corrected Wilcoxon signed rank tests, $\mathrm{p}_{\text {corr }}<0.05$ for significance; threshold indicated as a horizontal red dashed line). (E) Significance matrices comparing, per frequency band, z- 
1200 normalized iCoh values across different depths. Each cell $(i, j)$ in a matrix depicts the log-scaled

$1201 \quad$ porr obtained from statistically comparing coherence at channels with depths $i$ and $j$ in the AC.

1202 Red contour lines delimit regions of statistical significance (FDR-corrected Wilcoxon signed

1203 rank tests, significance when $\left.\mathrm{p}_{\text {corr }}<0.05\right)$. 
A

B

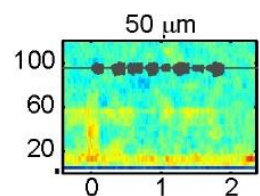

C

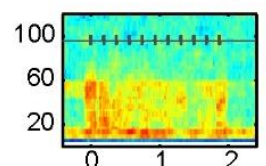

D
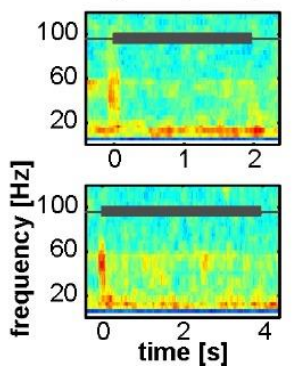
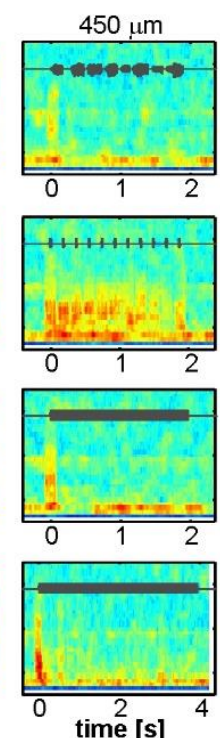
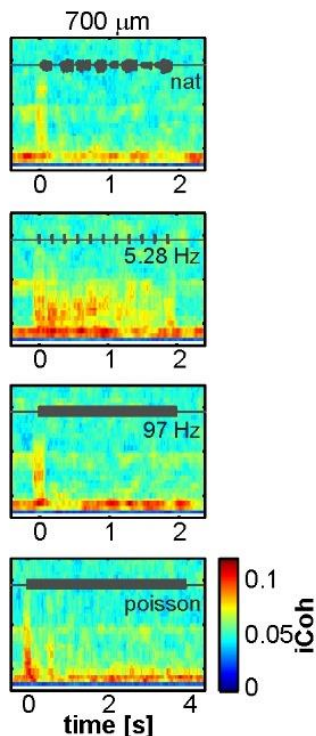

E

$\mathbf{F}$

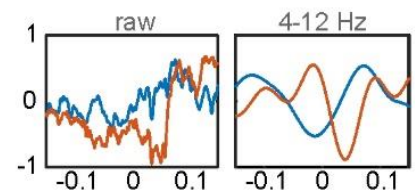

$25-45 \mathrm{~Hz}$

G
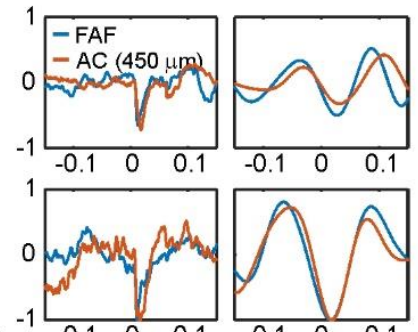

$\mathrm{H}$

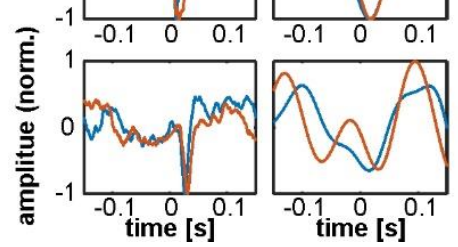

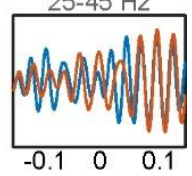
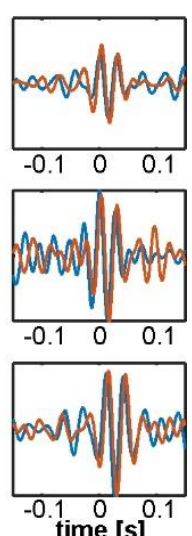

Fig. 4. Interareal phase synchrony during acoustic sequence processing. (A-D) Mean time-

resolved coherence between LFPs from the FAF and the AC at three representative depths (50, 450 and $700 \mu \mathrm{m})$, in response to the natural sequence $(\mathbf{A})$, a syllabic train of $5.28 \mathrm{~Hz}(\mathbf{B})$, a syllabic train of $97 \mathrm{~Hz}(\mathbf{C})$, and the syllabic train with a Poisson structure (D). Note that low frequency synchrony is high even without acoustic stimulation, and the appearance of gammaband evoked synchronization at the stimulus onset (time 0), albeit more weakly in response to the natural call in A. (E-H) LFP recordings from the AC (orange) and FAF (blue) around the time of stimulus onset (at 0 s; order in $\mathbf{E}-\mathbf{H}$ corresponds to order in A-D), from single trials in a representative penetration. Left column depicts the raw LFP, whereas middle and right columns depict field-potentials filtered in 4-12 and 25-45 Hz low-frequency and gamma-bands, respectively. 
A

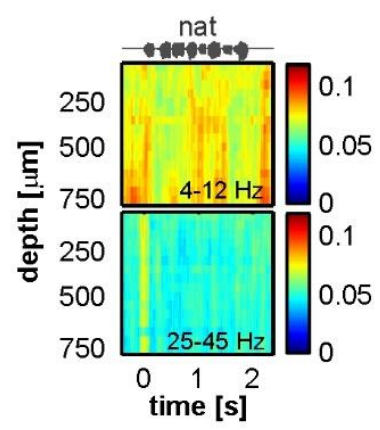

D

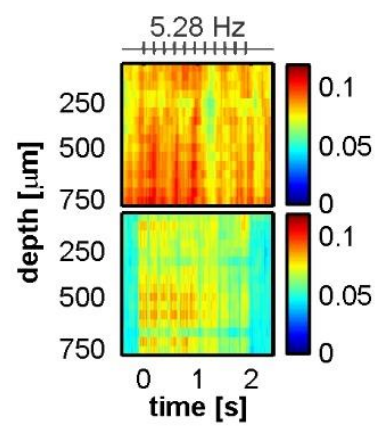

G

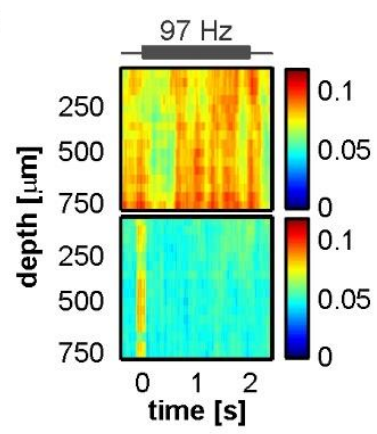

$\mathbf{J}$

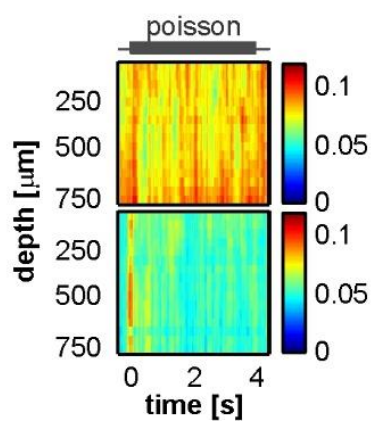

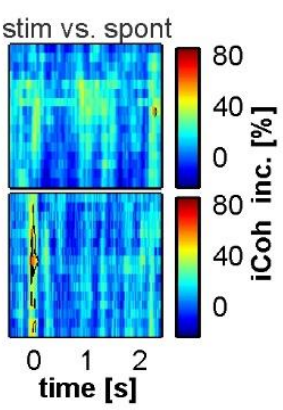
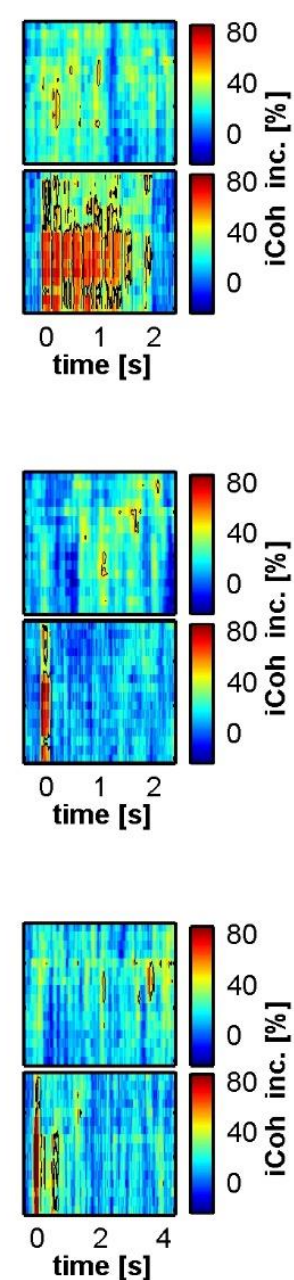

B

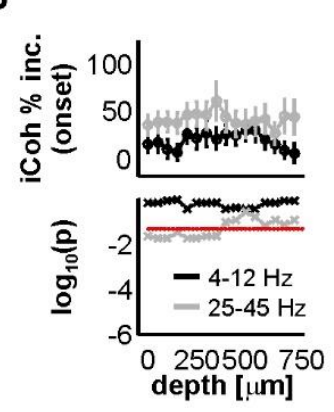

E

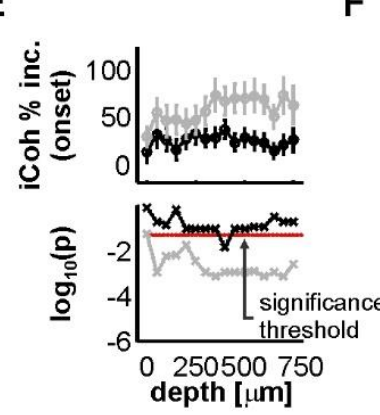

H

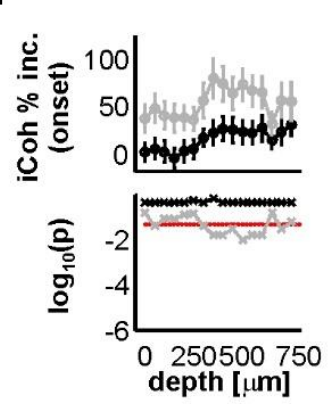

K

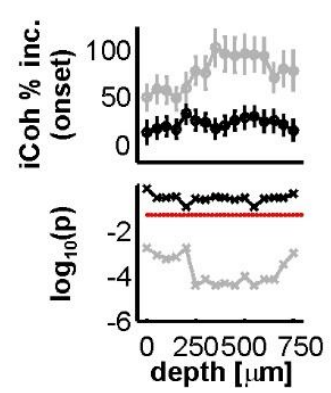

C

$\mathbf{F}$

I

$\mathbf{L}$
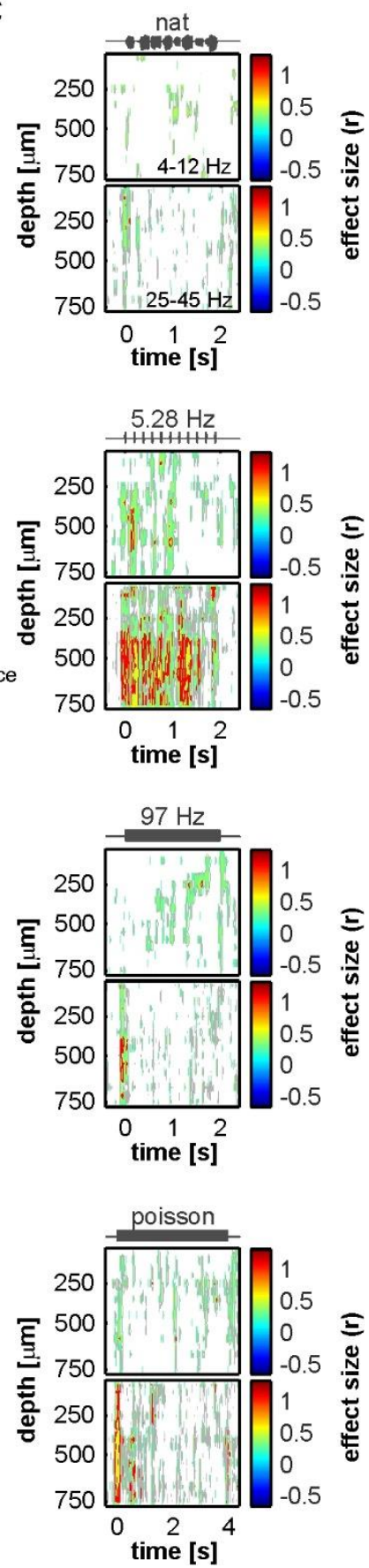

1219 Fig. 5. Acoustic stimulation alters FAF-AC coherence mostly in the gamma-band. (A) Top:

average time course of iCoh while animals listened to the natural sequence (left) across recording depths for low frequencies $(4-12 \mathrm{~Hz})$, and percentage increase of coherence in that range relative to the spontaneous activity (right). Bottom: same as Top, but for iCoh values in the gamma range $(25-45 \mathrm{~Hz})$. Black contour lines delimit regions with average increase of coherence $>50 \%$. (B) Population onset-related iCoh increase (median in the period of 0-150 ms after stimulus onset) across depths in the AC (black traces, low-frequency band iCoh; grey traces, 
1226 gamma-band iCoh; shown as mean +- SEM). On the bottom subpanel, log-scaled corrected p 1227 values obtained after testing that such increase was significantly different from $0 \%$ (black, low-

1228 frequency band; grey, gamma-band; FDR-corrected Wilcoxon signed rank tests, significance 1229 when $\mathrm{p}_{\mathrm{corr}}<0.05$, indicated as a red dashed line). (C) Time-resolved effect size of population 1230 iCoh percentage increase (r; see Methods) for the low-frequency band (top) and the gamma1231 range (bottom). Grey contour lines delimit regions of $\mathrm{r}>0.3$, whereas red contours mark regions 1232 of $\mathrm{r}>0.5$ (medium and large effect sizes, respectively). $\mathrm{r}$ values are only shown for time points, 1233 across channels, where the coherence increase was significantly higher than 0\% (Wilcoxon 1234 signed rank test, $\mathrm{p}<0.05)$. (D-F) Same as A-C but considering a syllabic train at $5.28 \mathrm{~Hz}$ as 1235 stimulus. (G-I) Same as A-C, the stimulus being a syllabic train at $97 \mathrm{~Hz}$. (J-L) Same as A-C, 1236 except the stimulus was the Poisson syllabic train. 
A

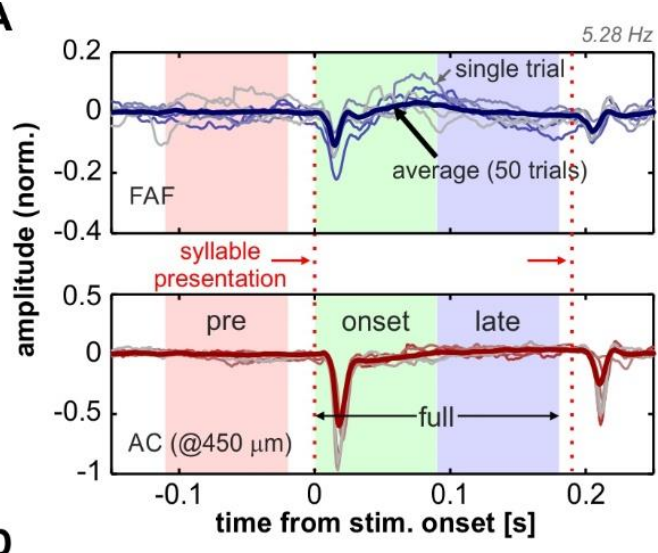

D

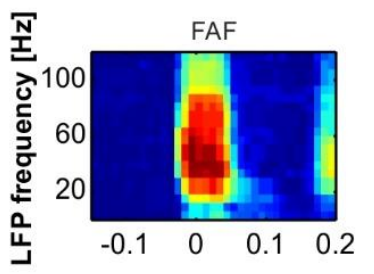

B
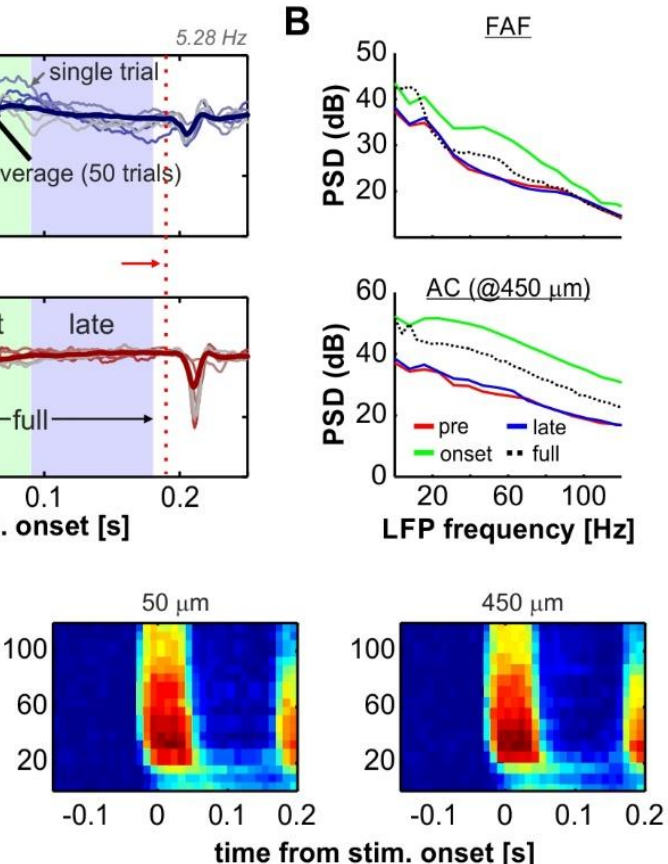

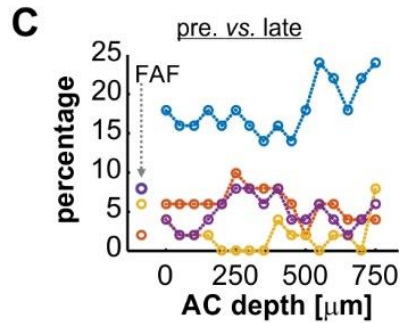

- low freq. $(0-15 \mathrm{~Hz})$

- low gamma $(25-45 \mathrm{~Hz})$

- high gamma $(45-80 \mathrm{~Hz})$

- broad gamma $(25-80 \mathrm{~Hz})$

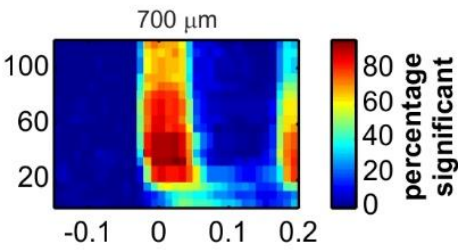

Fig. 6. Onset related power increase in AC and FAF. (A) Representative LFP recordings of one penetration pair (FAF, top; AC at $450 \mu \mathrm{m}$, bottom), depicting 6 single trials for illustrative purposes (thin lines) and the average across all 50 trials. The time segments of pre, onset, late, and full, used for analyses (see main text), are indicated in the graphs. First and second syllable presentations of the $5.28 \mathrm{~Hz}$ train are indicated with vertical, red dashed lines. (B) Power spectral density (PSD) of the pre (red), onset (green), late (blue) and full (black, dashed) periods from the data depicted in A (average over the 50 trials), in the FAF (top) and AC (bottom). (C) Percentage of penetrations (after a total of 50) for which the power (at several frequency bands, indicated in the figure) was significantly different during the late period than during the pre period. (D) Time-frequency analysis illustrating the percentage of penetrations in FAF and AC (at three representative depths: 50, 450 and $700 \mu \mathrm{m}$ ) in which the power at a given time window was significantly higher than the power at a window preceding the stimulus onset. 
A

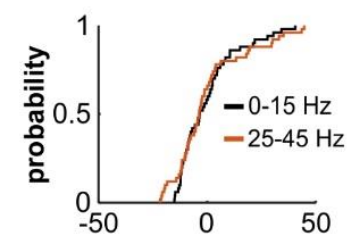

E

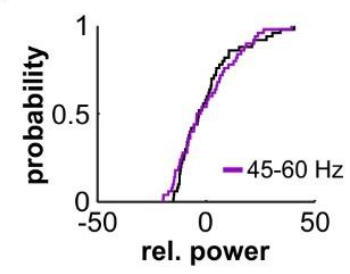

B

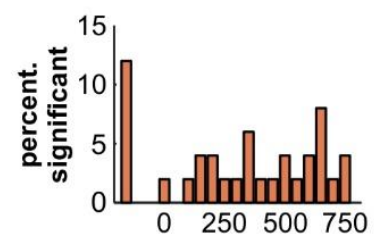

$\mathbf{F}$

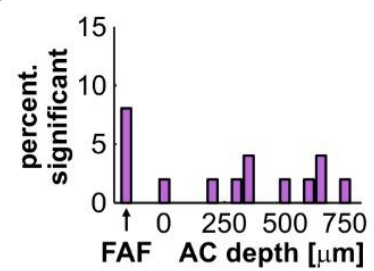

C

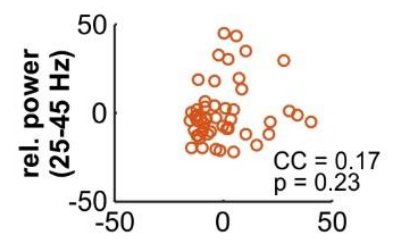

G

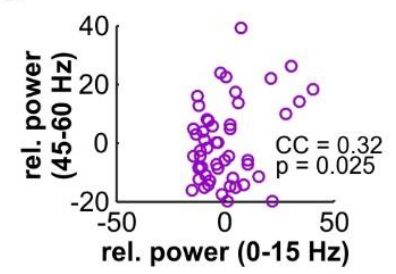

D

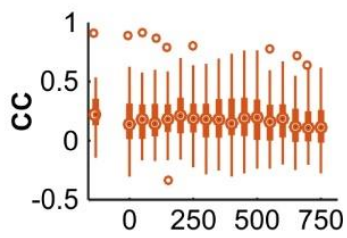

H

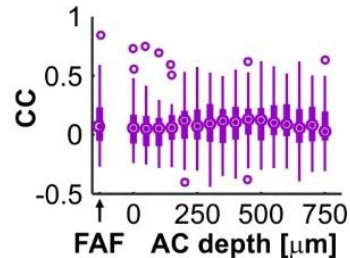

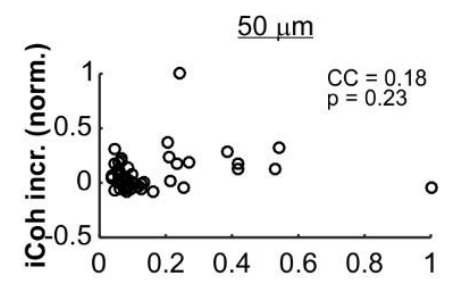

Fig. 7. Power distributions of gamma-band and low-frequency LFPs in AC and FAF. (A)

Distributions of the relative power of low-frequency $(0-15 \mathrm{~Hz}$; black) and gamma-band activity (25-45 Hz; orange) across trials, recorded from a single representative penetration in the FAF. These distributions were not significantly different from each other (2-sample KolmogorovSmirnov test, $\mathrm{p}=0.84)$. (B) Percentage from the total number of penetrations $(\mathrm{n}=50)$ for which the distributions of low-frequency and gamma $(25-45 \mathrm{~Hz})$ power were significantly different from each other at an alpha of 0.01, in FAF and at different depths of the AC. (C) Scatter plot and correlation coefficient (CC) of the trial-by-trial relationship between low-frequency and gamma band $(25-45 \mathrm{~Hz})$ power $(\mathrm{n}=50$ trials $)$, for the same representative penetration shown in A. The CC was of 0.17, and it was not significant: $\mathrm{p}=0.23$. (D) Distribution, in FAF and at all AC depths, of CCs between gamma-band $(25-45 \mathrm{~Hz})$ and low-frequency power. The median in the AC across depths was of 0.17, whereas the median in the FAF was of 0.22. (E-H) Similar to A-D, but the gamma range considered was of 45-60 Hz (signaled in purple). In panel E, both distributions were also not significant from each other (2-sample Kolmogorov-Smirnov test, $\mathrm{p}=$ 0.84). The $\mathrm{CC}$ in $\mathbf{G}$ was of 0.32 , and it was not significant at an alpha of 0.01 ( $\mathrm{p}=0.025)$. In panel $\mathbf{H}$, the median across depths in the $\mathrm{AC}$ was of 0.08 , whereas the median $\mathrm{CC}$ in the FAF was of 0.07. (I) Correlations between evoked-potential (ERP) energy in AC and gamma-band coherence increase (same as in Fig. 5) for three representative depths in the AC (at 50, 450 and 
bioRxiv preprint doi: https://doi.org/10.1101/2019.12.23.886770; this version posted February 25, 2020. The copyright holder for this preprint (which was not certified by peer review) is the author/funder, who has granted bioRxiv a license to display the preprint in perpetuity. It is made available under aCC-BY-NC-ND 4.0 International FPosfto-temporal coupling in bats

$1271 \quad 700 \mu \mathrm{m}$; all depths are shown in Fig. S3). Values are normalized for clarity. There were no 1272 significant correlations at any of the depths shown $(\mathrm{p}>=0.2)$.

1273 
A

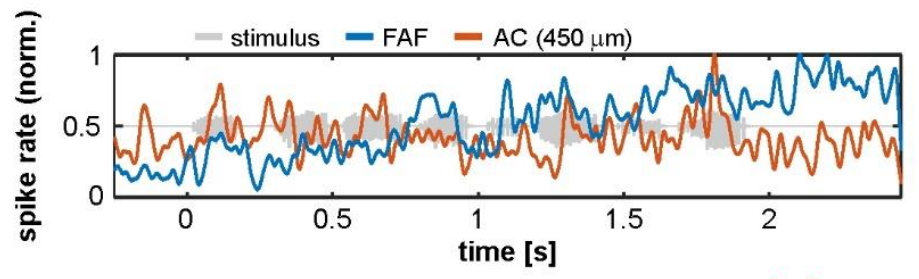

B

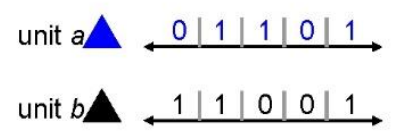

joint $(a, b) \underset{4 \mathrm{~ms}}{\stackrel{0,1|1,1| 1,0}{\leftrightarrows} 0,0 \mid 1,1}$
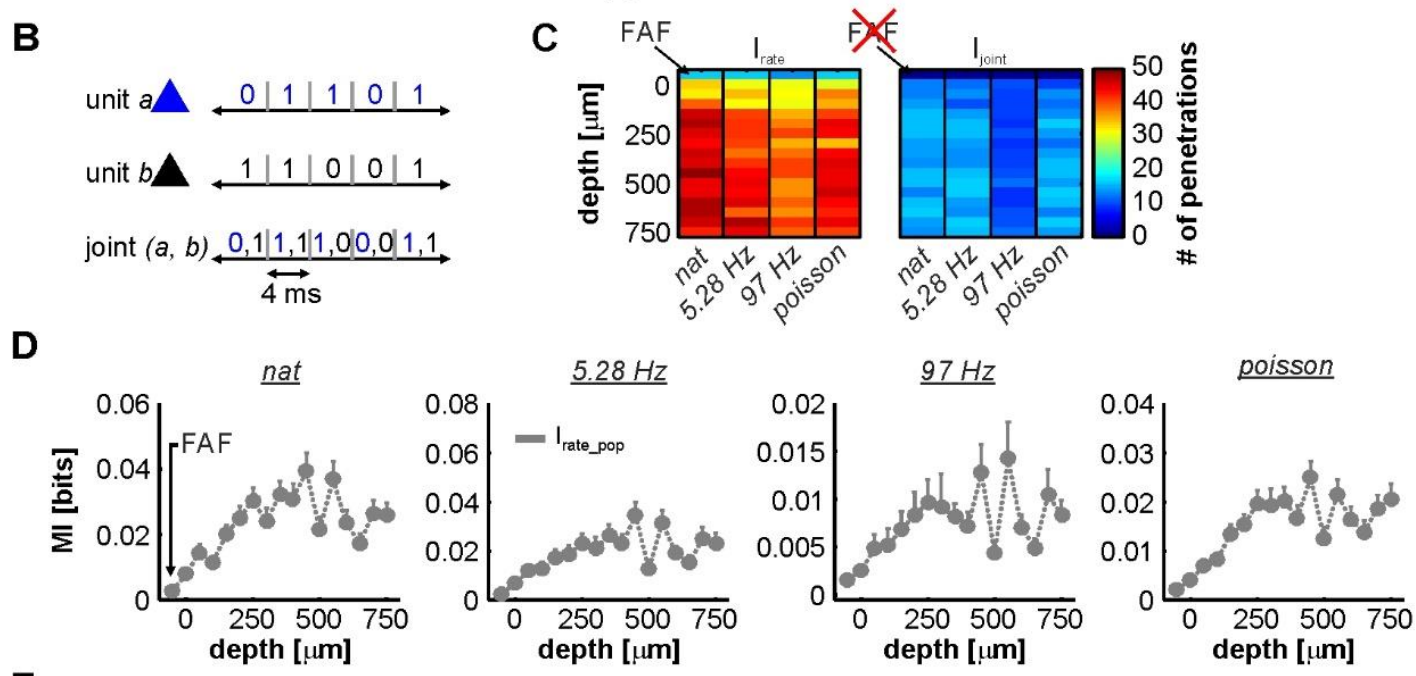

$\mathbf{E}$
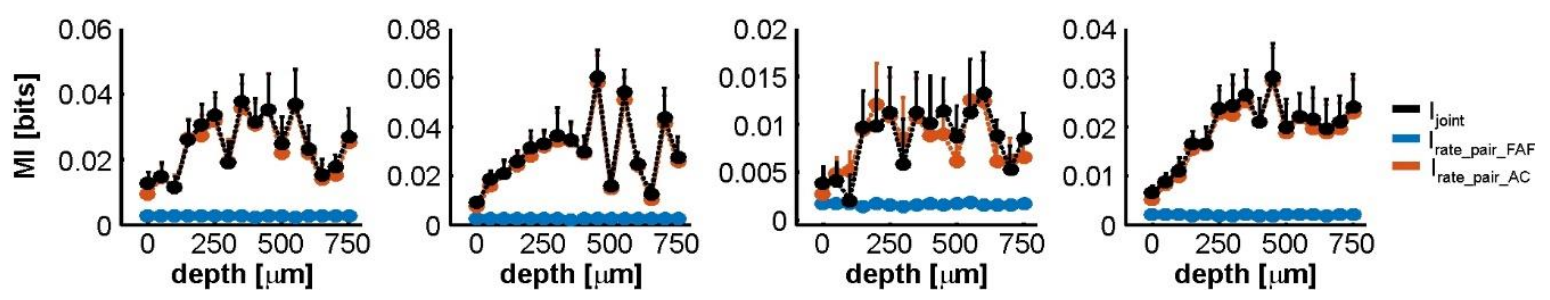

Fig. 8. Information in rate codes of the FAF-AC circuit. (A) Spiking activity in an exemplary simultaneous recording from the FAF (blue) and the AC (at $450 \mu \mathrm{m}$, red). Note the difference in the response patterns between the two structures. (B) Schematic representation of the joint code used for information theoretic calculations. (C) Number of units used to calculate $I_{\text {rate }}$ (left heatmap; 1 unit per penetration), for each stimulus (natural call, syllable train at $5.28 \mathrm{~Hz}$, syllable train at $97 \mathrm{~Hz}$, and Poisson syllable train) and depth in the $\mathrm{AC}$ (note that the first row in the heatmap corresponds to the FAF). Only units that had at least $0.1 \mathrm{bit} / \mathrm{s}$ of information were considered. The right heatmap depicts similar information, showing the number of pairs (FAF-AC spiking; 1 pair per penetration) used to calculate $\mathrm{I}_{\text {rate }}$ per stimulus and AC depth. In this case, FAF is missing because it is already part of each a pair. (D) Population $\mathrm{I}_{\text {rate }}$ in the FAF and across AC depths (note that the leftmost values correspond to the FAF), for each of the four stimuli presented (left to right). (E) $\mathrm{I}_{\text {joint }}$ (black) shown together with $\mathrm{I}_{\text {rate }}$ from AC (orange) and FAF (blue) units that conformed the pairs, across stimuli (i.e. Irate_pair_AC and Irate_pair_FAF). There were no consistent significant 
bioRxiv preprint doi: https://doi.org/10.1101/2019.12.23.886770; this version posted February 25, 2020. The copyright holder for this preprint (which was not certified by peer review) is the author/funder, who has granted bioRxiv a license to display the preprint in perpetuity. It is made available under aCC-BY-NC-ND 4.0 International FPosito-temporal coupling in bats

1288 differences between $\mathrm{I}_{\text {joint }}$ and $\mathrm{I}_{\text {rate, }}$ when the latter was calculated for AC units (Wilcoxon signed 1289 rank tests, $\mathrm{p}>0.05)$. 
A

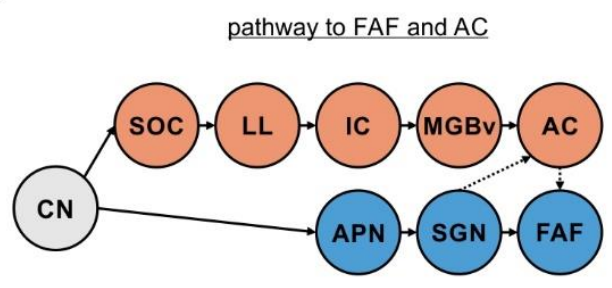

D

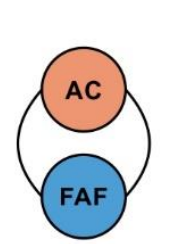

FAF-AC spont. low-freq. coherence
B

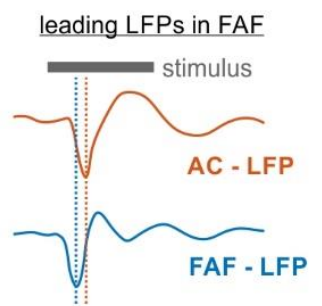

C

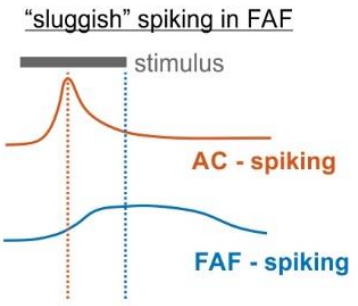

E
$\mathbf{F}$

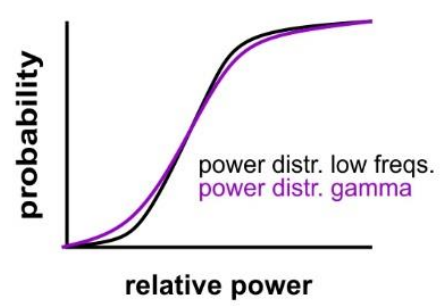

G

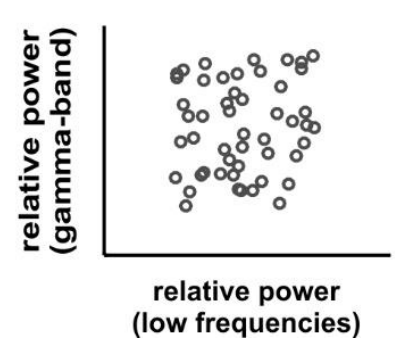

FAF-AC auditory-evoked gamma coherence

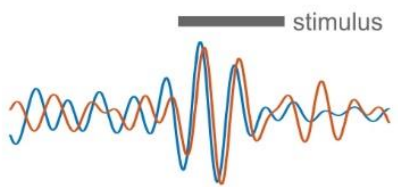

H

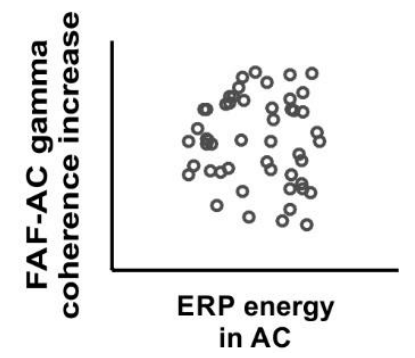

Fig. 9. Functional coupling dynamics in the FAF-AC circuit of $C$. perspicillata. (A) Representation of the auditory pathway to the FAF and the AC $(\mathrm{CN}$, cochlear nucleus; SOC, superior olivary complex; LL, lateral lemniscus; IC, inferior colliculus; MGBv, ventral division of the medial geniculate body of the thalamus; AC, auditory cortex; APN, anterolateral periolivary nucleus; SGN, suprageniculate nucleus of the thalamus; FAF, frontal auditory field). (B) Schematic representation illustrating that stimulus-related LFPs in FAF lead relative to those in the AC (see Fig. 1). (C) Fast LFP responses in FAF do not necessarily elicit fast spiking responses (schematic). Neurons in the frontal region typically respond more "sluggishly" than their auditory cortical counterparts. (D) FAF and AC were coherent in low-frequencies during spontaneous activity (i.e. in the absence of sound stimulation). (E) During acoustic processing in passive listening animals, low-frequency coherence was unaltered in the FAF-AC circuit, although there was an emergence of auditory-evoked gamma band coherence in the network. Traces in panels D and E are based on data shown in Figs. 3 and 4. Note that, for illustrative purposes, the temporal scales and amplitudes in $\mathbf{D}$ and $\mathbf{E}$ are not comparable. (F) Distributions of power in low and gamma-band frequencies were typically not significantly different from each other. However, there was very weak trial-bytrial correlation between low-frequency and gamma power $(\mathbf{G})$, as well as very low correlations 
bioRxiv preprint doi: https://doi.org/10.1101/2019.12.23.886770; this version posted February 25, 2020. The copyright holder for this preprint

(which was not certified by peer review) is the author/funder, who has granted bioRxiv a license to display the preprint in perpetuity. It is made available under aCC-BY-NC-ND 4.0 International FPôfto-temporal coupling in bats

1308 between event-related potential (ERP) energy and gamma coherence increase across penetrations $1309 \quad(\mathbf{H})$.

1310

1311 